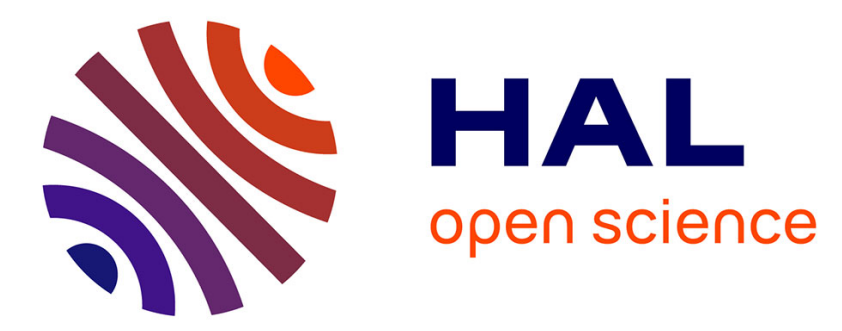

\title{
Proper orthogonal decomposition analysis and modelling of large-scale flow reorientations in a cubic Rayleigh-Bénard cell
}

Laurent Soucasse, Berengere Podvin, Philippe Rivière, Anouar Soufiani

\section{- To cite this version:}

Laurent Soucasse, Berengere Podvin, Philippe Rivière, Anouar Soufiani. Proper orthogonal decomposition analysis and modelling of large-scale flow reorientations in a cubic Rayleigh-Bénard cell. Journal of Fluid Mechanics, 2019, 881, pp.23-50. 10.1017/jfm.2019.746 . hal-02342436

\section{HAL Id: hal-02342436 \\ https://hal.science/hal-02342436}

Submitted on 31 Oct 2019

HAL is a multi-disciplinary open access archive for the deposit and dissemination of scientific research documents, whether they are published or not. The documents may come from teaching and research institutions in France or abroad, or from public or private research centers.
L'archive ouverte pluridisciplinaire HAL, est destinée au dépôt et à la diffusion de documents scientifiques de niveau recherche, publiés ou non, émanant des établissements d'enseignement et de recherche français ou étrangers, des laboratoires publics ou privés. 


\title{
Proper Orthogonal Decomposition analysis and modelling of large-scale flow reorientations in a cubic Rayleigh-Bénard cell
}

\author{
Laurent Soucasse $^{1} \dagger$, Bérengère Podvin ${ }^{2}$, Philippe Rivière ${ }^{1}$ and \\ Anouar Soufiani ${ }^{1}$ \\ ${ }^{1}$ Laboratoire EM2C, CNRS, CentraleSupélec, Université Paris-Saclay, 91192 Gif-sur-Yvette, \\ France \\ ${ }^{2}$ LIMSI-CNRS, rue Von Neumann, Orsay, 91403 CEDEX, France
}

(Received xx; revised xx; accepted xx)

This paper investigates the large-scale flow reorientations of Rayleigh-Bénard convection in a cubic cell using Proper Orthogonal Decomposition (POD) analysis and modelling. A Direct Numerical Simulation (DNS) is performed for air at a Rayleigh number of $10^{7}$ and shows that the flow is characterized by four quasi-stable states, corresponding to a Large-Scale Circulation (LSC) lying in one of the two diagonal planes of the cube with a clockwise or anticlockwise motion, with occasional brief reorientations. POD is applied to the joint velocity and temperature fields of an enriched database which captures the statistical symmetries of the flow. We found that each quasi-stable state consists of a superposition of four spatial modes representing three types of structures: (i) a meanflow mode consisting of two stacked counter-rotating torus-like structures; (ii) two largescale 2D-rolls (pair of degenerated modes) which form large-scale diagonal-rolls when combined together; (iii) an eight-roll mode that transports fluid from one corner to the other and strengthens the circulation along the diagonal. In addition, we identified three other modes that play a role in the reorientation process: two boundary-layer modes (pair of degenerated modes) that connect the core region with the horizontal boundary layers and one mode associated with corner rolls. The symmetries of the different POD modes are discussed, as well as their temporal dynamics. A description of the reorientation process in terms of POD modes is provided and compared with other modal approaches available in the literature. Finally, Galerkin projection is used to derive a POD-based reduced order model. Unresolved modes are accounted for in the model by an extra dissipation term and the addition of noise. A seven-mode model is able to reproduce the low frequency dynamics of the large-scale reorientations as well as the high frequency dynamics associated with the LSC rotation. Linear stability analysis and sensitivity analysis confirm the role of the boundary-layer modes and the corner-rolls mode in the reorientation process.

Key words: cubic Rayleigh-Bénard cell, flow reorientation, Proper Othogonal Decomposition

$\dagger$ Email address for correspondence: laurent.soucasse@centralesupelec.fr 


\section{Introduction}

Turbulent buoyancy-driven convection is an ubiquitous phenomenon, occurring in the atmosphere or the ocean and controlling various engineering applications. RayleighBénard convection is a simple configuration for studying such processes, in which a fluid is confined between two horizontal plates, heated from the bottom and cooled from above. One of the main features of interest of the convective transport is the large-scale motion from bottom hot to top cold walls, because it carries most of the kinetic energy and most of the heat. The structure of this large-scale motion, referred to as Large Scale Circulation (LSC) in the literature, strongly depends on the shape of the container. It is made of one or several rolls depending on the ratio between the vertical and the horizontal characteristic lengths. The position and the shape of these large-scale coherent structures may vary over time and are affected by the small scale properties of the turbulence.

In a cylindrical container with aspect ratio of unity (diameter equal to height), the LSC is made of a single dominant roll lying in a vertical square section. The LSC experiences irregular azimuthal displacements called reorientations, consisting of either a rotation of the LSC around the centreline or a cessation of the LSC (Brown et al. 2005; Benzi \& Verzicco 2008; Mishra et al. 2011). These reorientation events follow a Poisson distribution over a wide range of time scales (Sreenivasan et al. 2002; Brown et al. 2005). A specific effort has been devoted to discriminating the physical mechanisms beyond rotation-led and cessation-led reorientations. The latter phenomenon is often reproduced using quasi-square experimental devices (Sugiyama et al. 2010) or performing 2D numerical simulations in a square Rayleigh-Bénard cell (Castillo-Castellanos et al. 2016). The growth of counter-rotating corner rolls is shown to be responsible for the cessation and reorientation of the LSC in this configuration.

The study of the large-scale motion of turbulent natural convection in cubic RayleighBénard cells has received only a recent interest compared to the cylindrical or square configurations. Unlike the cylindrical geometry, the cubic container does not possess an azimuthal symmetry and the LSC stabilizes in one of the two diagonal vertical planes leading to four potential quasi-stable states for the flow (the two diagonal planes combined with clockwise or anticlockwise motion). Random low-frequency reorientations between these quasi-stable states have been observed in experiments (Vasiliev et al. 2016) and numerical simulations (Foroozani et al. 2017) which consist in a rotation of the LSC from one diagonal plane to the other. No cessation-led reorientation events have been reported yet, although the study of Giannakis et al. (2018) suggests that reversals (reorientations of an angle $\pi$ ) can happen.

Several models have been proposed to explain the dynamics of the reorientations in the cube. Bai et al. (2016) derived a low-order model for predicting the time evolution of the orientation angle $\theta$ of the LSC in a horizontal plane. They extended a previous model predicting the reorientations in the cylinder (Brown \& Ahlers 2007) by adding a potential term corresponding to the pressure of the sidewalls. This potential is minimum when the LSC lies in the diagonal planes (when the horizontal characteristic length of the LSC is the heighest) and drives the orientation angle towards one of the four corners of a horizontal cross section which corresponds to the four quasi-stable states. A Gaussian stochastic noise is injected in the equation for modelling the turbulent fluctuations and reorientations from one corner to another occur when a fluctuation is high enough to cross the potential barrier corresponding to a mid-square vertical plane.

Another mechanism for explaining flow reorientations has been suggested by Vasiliev et al. (2018). These authors considered the diagonal LSC rolls as the superposition of two large scale $2 \mathrm{D}$ rolls lying in the two orthogonal vertical mid-planes of the cube with 
clockwise or anticlockwise motion. A reorientation event is understood as the cessation and the reversal of one of these two planar rolls. Following previous works in the square cell (Chandra \& Verma 2011, 2013), they modelled the 3D velocity field by superposing a two-modes description (a single-roll mode and a quadrupolar mode) for each of the planar flows. They performed a Fourier analysis of this model flow which compares well with numerical simulation data.

Giannakis et al. (2018) performed a Koopman analysis of direct numerical simulation data in a cubic Rayleigh-Bénard cell. The Koopman analysis offers a modal description of the velocity and temperature fields in terms of eigenmodes that evolve periodically in time according to their complex eigenvalue (Rowley et al. 2009). The authors extracted four primary Koopman eigenmodes which were necessary to reconstruct the four quasistable macroscopic states. Two of these primary modes are a complex conjugate pair and correspond to single-roll modes lying in the two diagonal planes. Eight secondary Koopman eigenfunctions (four complex conjugate pairs) were also identified and each pair was shown to be associated with one of the quasi-stable states. The analysis was however limited by the time integration (though very large for a DNS) because of the lowfrequency of the reorientation events. Especially the four stable states were not equally sampled and the Koopman eigenfunctions did not follow expected symmetry properties.

Unlike the Koopman analysis, the Proper Orthogonal Decomposition (POD) is a modal decomposition method which extracts pure spatial modes from a statistical analysis of sampled numerical data without any assumption about a temporal connection between the different samples. The method is efficient in capturing the large-scale coherent structures as POD modes are selected using an energy-based criterion. In addition, POD-based low order models can be easily derived using Galerkin projection of the Navier-Stokes equations onto a reduced set of POD modes, since they form an orthogonal basis. POD has been applied to Rayleigh-Bénard convection in cylindrical configuration (Bailon-Cuba et al. 2010) or in rectangular cavities (Verdoold et al. 2009; Podvin \& Sergent 2012). In the square cell, the POD analysis has provided a better understanding of the reversal mechanism and has led to the development of low order models, able to reproduce the dynamics of the large-scale motion (Podvin \& Sergent 2015, 2017). However, the method has not been applied to study the reorientation mechanisms in the cubic cell so far.

This paper aims at giving a novel insight into the large-scale flow reorientations in the cubic Rayleigh-Bénard cell from POD analysis and modelling. We have performed a long term Direct Numerical Simulation (DNS) of temperature and velocity fields at a Rayleigh number of $10^{7}$ in air at room temperature (Prandtl number of 0.707 ) whose results are given in section 2. Section 3 discusses in detail the empirical POD spatial modes extracted from the simulation in light of their role in the reorientation process. A 6 modes reduced order model that captures the dynamics of the large-scale reorientations is then derived in section 4 .

\section{Direct numerical simulations}

\subsection{Problem setup and governing equations}

We consider a cubical cavity of size $L$ filled with air at ambient temperature, heated from below and cooled from above. Top and bottom walls are maintained at uniform temperature $T_{c}$ and $T_{h}$ and the four lateral walls are assumed to be adiabatic. Governing equations under Boussinesq approximation are

$$
\boldsymbol{\nabla} \cdot \boldsymbol{u}=0
$$




$$
\begin{gathered}
\frac{\partial \boldsymbol{u}}{\partial t}+\boldsymbol{u} \cdot \boldsymbol{\nabla} \boldsymbol{u}=-\nabla p+\operatorname{Pr} \theta \boldsymbol{e}_{\boldsymbol{z}}+\frac{\operatorname{Pr}}{\sqrt{R a}} \nabla^{2} \boldsymbol{u}, \\
\frac{\partial \theta}{\partial t}+\boldsymbol{u} \cdot \boldsymbol{\nabla} \theta=\frac{1}{\sqrt{R a}} \nabla^{2} \theta
\end{gathered}
$$

where $\boldsymbol{u}=(u, v, w)$ is the dimensionless velocity vector, $p$ is the dimensionless pressure and $\theta$ is the reduced temperature. A no-slip velocity condition $(\boldsymbol{u}=0)$ is prescribed on the six walls of the cavity and thermal boundary conditions are written as follows

$$
\left.\begin{array}{l}
\theta=0.5 \quad \text { on } \quad z=0, \\
\theta=-0.5 \quad \text { on } \quad z=1, \\
\boldsymbol{\nabla} \theta \cdot \boldsymbol{n}=0 \quad \text { on } \quad x=0, x=1, y=0, y=1
\end{array}\right\}
$$

where $x, y$ and $z$ are the dimensionless Cartesian coordinates. The Rayleigh number which controls the flow regime is set to $10^{7}$ and is given by $R a=g \beta_{e} \Delta T L^{3} /(\nu \kappa)$, where $g$ is the gravitational acceleration, $\beta_{e}$ is the thermal expansion coefficient, $\nu$ is the kinematic viscosity, $\kappa$ is the thermal diffusivity and $\Delta T=\left(T_{h}-T_{c}\right)$ the temperature difference between hot and cold walls. The Prandtl number is set to 0.707 and is given by $\operatorname{Pr}=\nu / \kappa$. Equations are made dimensionless using the length of the cavity $L$, the reference time $L^{2} /(\kappa \sqrt{R a})$ and the reduced temperature $\theta=\left(T-T_{0}\right) / \Delta T, T_{0}$ being the mean temperature between hot and cold walls.

The problem satisfies four independent reflection symmetries $S_{x}, S_{y}, S_{z}$ and $S_{d}$ with respect to the planes $x=0.5, y=0.5, z=0.5$ and $x=y$ (Puigjaner et al. 2008). These symmetries act on the velocity and temperature fields as follows

$$
\left.\begin{array}{rl}
S_{x}: \begin{array}{l}
(x, y, z) \rightarrow(1-x, y, z) \\
(u, v, w, \theta) \rightarrow(-u, v, w, \theta)
\end{array}
\end{array}\right\}
$$

These four elementary symmetries generate a symmetry group of sixteen elements. In unsteady regime, we expect these symmetries to be satisfied by the time-averaged flow field.

\subsection{Numerical methods}

Equations (2.1-2.3) are solved using a Chebyshev collocation method for the three dimensions of space (Xin \& Le Quéré 2002). The pressure-flow coupling is ensured by a projection method, in two steps. First, momentum and energy equations are solved using the previous time step pressure field. Then, a pressure correction term is calculated from a Poisson equation and the predicted velocity is corrected in order to force the velocity divergence free condition. Time integration is performed through a second order temporal scheme combining a Backward Differentiation (BDF2) scheme for the linear terms with an Adams Bashforth extrapolation of convective terms. This algorithm is implemented for parallel computations applying domain decomposition along the $z$-vertical direction (Xin et al. 2008). 

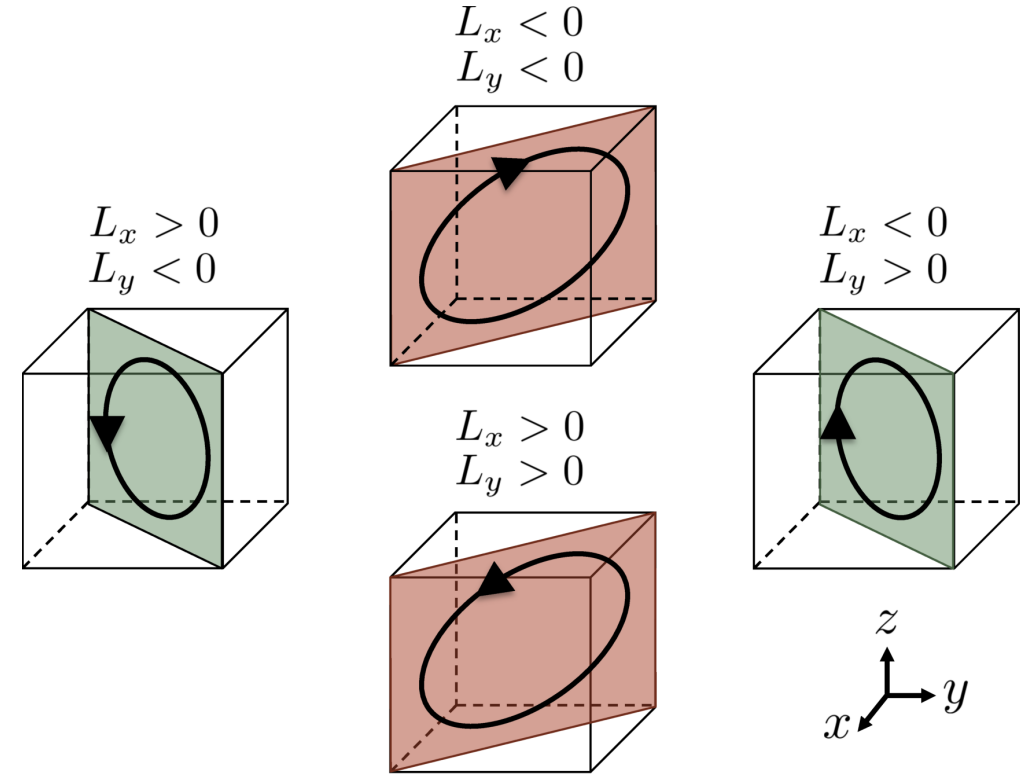

FiguRE 1. Diagram of the four quasi-stable flow states and associated sign of $x$ and $y$ components of the global angular momentum.

The spatial mesh is made of 81 Chebyshev collocation points in the $x$ and $y$ directions and $4 \times 21$ Chebyshev collocation points ( 4 spatial domains discretized with 21 points) in the $z$ direction. The number of spatial collocation points in the kinetic and thermal boundary layers $\left(N_{u, B L}\right.$ and $\left.N_{\theta, B L}\right)$ is about 10 in the $x$ and $y$ directions and about 6 in the $z$ direction, which is satisfactory regarding the criterion proposed by Shishkina et al. (2010) $\left(N_{u, B L}=0.31 R a^{0.15}\right.$ and $N_{\theta, B L}=0.35 R a^{0.15}$ for $\left.\operatorname{Pr} \simeq 0.7\right)$. The thicknesses of the kinetic and thermal boundary layers have been estimated using the correlations provided by the same authors and are respectively equal to $\delta_{u} / L=0.027$ and $\delta_{\theta} / L=0.031$ at $R a=10^{7}$.

The dimensionless time step is $2.5 \times 10^{-3}$. Starting from a fluid at $\boldsymbol{u}=\mathbf{0}$ and $\theta=0$, equations are first integrated over a dimensionless time period of 2,000 in order to reach the asymptotic regime and then integrated over a dimensionless time period of 10,000 to conduct the analysis. The time-averaged Nusselt number over this second period is equal to 16.24 , which is consistent with the value of 16.57 found by Giannakis et al. (2018) for the same Rayleigh number and a nearly identical Prandtl number of 0.7 .

\subsection{Large-scale flow structures}

Four quasi-stable flow states are observed in the numerical simulation. The Large Scale Circulation (LSC) associated with these states consists of a dominant roll in one of the two diagonal planes $x=y$ or $x=1-y$ with a clockwise or anticlockwise motion as shown in figure 1. These four flow states have been reported by other researchers in numerical studies (Foroozani et al. 2017; Giannakis et al. 2018; Vasiliev et al. 2018) and experimental studies (Bai et al. 2016; Vasiliev et al. 2016)).

The switches between these states can be monitored with the time evolution of the $x$ and $y$ components of the global angular momentum with respect to the centre of the cavity $\boldsymbol{r}_{0}=(0.5 ; 0.5 ; 0.5)$

$$
\boldsymbol{L}=\int\left(\boldsymbol{r}-\boldsymbol{r}_{0}\right) \wedge \boldsymbol{u} d \boldsymbol{r}
$$




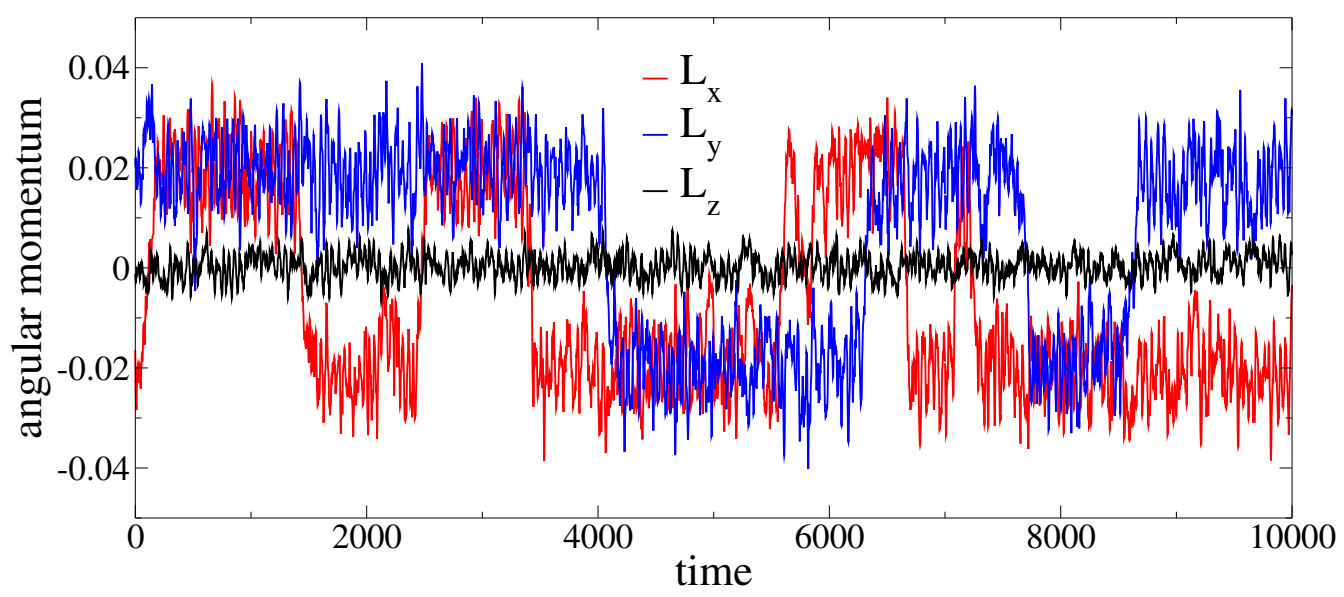

Figure 2. Time evolution of the three components of the angular momentum.

that is plotted in Fig. 2. Both components $L_{x}$ and $L_{y}$ display quasi-stable periods with moderate oscillations around a mean value of \pm 0.2 separated by abrupt aperiodic sign switches. A sign switch of one of the $x$ or $y$ components of the global angular momentum corresponds to a rotation of the LSC of $\pi / 2$ from one diagonal plane to another. Such transition will be referred to as a reorientation of the LSC in the remainder of the paper. Interestingly, a simultaneous sign switch of both $L_{x}$ and $L_{y}$ is never observed in the simulation. This would be equivalent to a flow reversal (as observed in a square Rayleigh-Bénard cell by Podvin \& Sergent (2015)) where the direction of rotation of the LSC changes within the same diagonal plane. While the switching mechanism between states is a non periodic phenomenon, the average time spent in a quasi-stable flow state can be estimated by tracking the sign switches of angular momentum components. About 12 switches were identified in the simulation, which corresponds to a mean period of about 830 units. A more systematic analysis will be carried out in the next section.

The structure of the flow in one of the quasi-stable states $\left(L_{x}<0\right.$ and $\left.L_{y}<0\right)$ can be observed in figure 3 where an instantaneous flow field at $t=4610$ and a timeaveraged flow-field during the period [4500-5500] are shown. A fluid parcel rotates in the diagonal LSC in approximately 50 time units if we take a reference velocity of 0.076 , obtained by averaging the absolute value of the vertical velocity in the horizontal midplane, and a reference travel path length of 3.85, estimated by an ellipsoid trajectory in the diagonal plane. This estimate agrees well with the dominant oscillatory time scale $T_{c} \sim 45$ identified in the temporal spectrum of the angular momentum. A period of 1000 time units is then sufficient to correctly capture the mean features of the quasistable state. The instantaneous and time-averaged flow fields in figure 3 show a LSC lying in the diagonal plane $x=1-y$. The fluid flows up along the left vertical edge $(x ; y)=(1 ; 0)$ and flows down along the right vertical edge $(x ; y)=(0 ; 1)$. This main diagonal roll is slightly tilted and two small counter-rotating rolls are noticeable in the top left corner $(x ; y ; z)=(1 ; 0 ; 1)$ and in the bottom right corner $(x ; y ; z)=(0 ; 1 ; 0)$. Along the isothermal walls, part of the fluid moves away from the main diagonal plane and forms secondary rolls in the opposite corners. These structures spread up to midheight and get back to the main stream along the two vertical edges of the diagonal plane. 

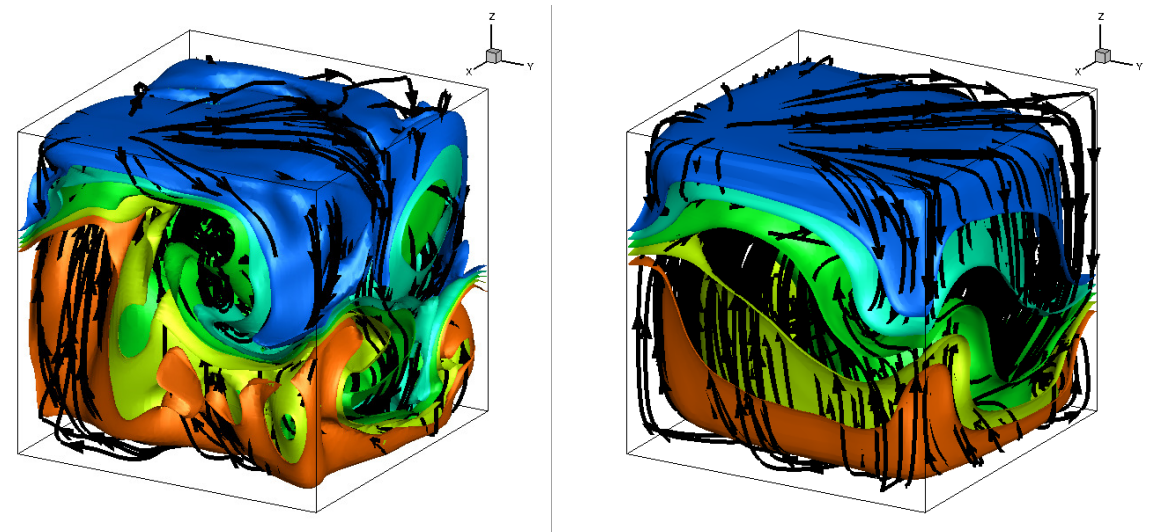

Figure 3. Instantaneous flow field at $t=4610$ (left) and time-averaged flow field during the period $t \in[4500-5500]$ (right). Streamlines and isotherms $\theta=\{0 ; \pm 0.05 ; \pm 0.1\}$.

\section{Proper Orthogonal Decomposition analysis}

\subsection{Methodology}

The Proper Orthogonal Decomposition (Berkooz et al. 1993) aims at finding an optimal basis of spatial modes $\phi_{n}(\boldsymbol{r})$ for a vector field $\boldsymbol{U}(\boldsymbol{r}, t)$ with respect to its energy content $\int\left\langle\boldsymbol{U}(\boldsymbol{r}, t)^{2}\right\rangle d \boldsymbol{r}$, where $\langle\cdot\rangle$ denotes the time average. The spatial modes are sought such that they maximize the projection of $\boldsymbol{U}(\boldsymbol{r}, t)$ which leads to the following eigenvalue problem

$$
\int_{\Omega} \sum_{k=1}^{M}\left\langle U^{m}(\boldsymbol{r}, t) U^{k}\left(\boldsymbol{r}^{\prime}, t\right)\right\rangle \phi_{n}^{k}\left(\boldsymbol{r}^{\prime}\right) d \boldsymbol{r}^{\prime}=\lambda_{n} \phi_{n}^{m}(\boldsymbol{r}),
$$

$M$ being the dimension of the vector field and $\Omega$ the spatial domain. The POD modes form an orthonormal basis allowing the decomposition of the variable field $\boldsymbol{U}(\boldsymbol{r}, t)$

$$
\boldsymbol{U}(\boldsymbol{r}, t)=\sum_{n=1}^{\infty} a_{n}(t) \boldsymbol{\phi}_{n}(\boldsymbol{r})
$$

The projection coefficients $a_{n}(t)$ are statistically uncorrelated and their energy is equal to the eigenvalue $\lambda_{n}:\left\langle a^{n}(t) a^{m}(t)\right\rangle=\delta_{n m} \lambda_{n}$ where $\delta_{n m}$ is the Kronecker symbol. The eigenvalue associated with a POD mode is thus a measure of its energy content and the objective of the method is to restrict the decomposition (3.2) to a few modes with the largest eigenvalues so that the flow dynamics can be analyzed in a low-order subspace able to capture the most of the energy of the field $\boldsymbol{U}(\boldsymbol{r}, t)$.

To apply the POD method to natural convection flows, we have considered vector fields $\boldsymbol{U}=[u, v, w, \gamma \theta]$ where $\gamma$ is a scaling factor given by

$$
\gamma^{2}=\left\langle\frac{\int\left[u^{2}(\boldsymbol{r}, t)+v^{2}(\boldsymbol{r}, t)+w^{2}(\boldsymbol{r}, t)\right] d \boldsymbol{r}}{\int \theta^{2}(\boldsymbol{r}, t) d \boldsymbol{r}}\right\rangle
$$

introduced for instance by Podvin \& Le Quéré (2001). This scaling factor allows the velocity and temperature fields to have the same energy and is equal to $\gamma=1.303$ in the present study. We have also applied the POD method independently to velocity fields and temperature fields: the same first spatial modes were obtained although they were not ranked in the same order in the energy spectrum. A similar conclusion was drawn by Podvin \& Sergent (2015) for natural convection flows in a square Rayleigh-Bénard cell. 
For solving the eigenvalue problem (3.1), the method of snapshots (Sirovich 1987) is used. It relies on the ansatz where the eigenmodes are written as a linear combination of $N$ instantaneous flow fields $\boldsymbol{U}\left(\boldsymbol{r}, t_{i}\right)$ (snapshots) extracted at discrete times $t_{i}$

$$
\phi_{n}(\boldsymbol{r})=\sum_{i=1}^{N} \boldsymbol{U}\left(\boldsymbol{r}, t_{i}\right) \Phi_{n}^{i} .
$$

The vectors $\boldsymbol{\Phi}_{n}$ satisfy the eigenvalue problem

$$
\boldsymbol{C} \boldsymbol{\Phi}_{n}=\lambda_{n} \boldsymbol{\Phi}_{n}
$$

where $\boldsymbol{C}$ is the temporal correlation matrix of the snapshots given by

$$
C_{i j}=\frac{1}{N} \int_{\Omega} \boldsymbol{U}\left(\boldsymbol{r}, t_{i}\right) \cdot \boldsymbol{U}\left(\boldsymbol{r}, t_{j}\right) d \boldsymbol{r} .
$$

Snapshots of the temperature and velocity fields are extracted from the DNS with a constant sampling period of 10 in dimensionless time units, making a statistical set of 1,000 samples. The four flow states described in section 2.3 are not equally represented in the snapshot set, although there is no physical evidence suggesting that these four states are not equiprobable. This is an artefact owing to the relatively short simulation time compared to the time scale separating two reorientations. In order to enforce an equal statistical weight for each flow state and to improve the convergence of the POD method, we have built an enlarged snapshot set, obtained by the action of the symmetry group of the problem on the original snapshot set. As seen in section 2.1 the symmetry group contains 16 elements and this allows us to multiply the number of snapshots by a factor of 16 to obtain a final snapshot set of 16,000 samples. The use of statistical symmetries of the flow in POD is discussed at length by Moin \& Moser (1989) and Holmes et al. (1996).

\subsection{POD spectrum and associated modes}

The POD eigenspectrum is shown in Fig. 4. The decay of the spectrum is rather slow owing to the three dimensions of space and the turbulent nature of the flow. The first three modes contain however $60 \%$ of the total energy. An interesting feature of the spectrum is the presence of pairs of degenerated modes with strictly identical eigenvalues, for instance modes 2 and 3, modes 5 and 6 or modes 10 and 11. These pairs of modes result from the equivalence between directions $x$ and $y$ in the horizontal plane and are associated with spatial structures that are the image of each other after a rotation of $\pi / 2$ around the $z$-axis. Interestingly, when applying POD to the original (non-symmetrised) snapshot set, we have identified similar pairs of structures, linked by a rotation of $\pi / 2$ around the z-axis. However one observes a small discrepancy in energy between these modes due to the statistical imbalance between the four quasi-stable flow states. Apart from the first three modes that clearly stand out from the spectrum, it is difficult to decide which modes have to be retained in a reduced decomposition based on an energy criterion. In the following we will restrict the analysis to the first seven modes. This will be justified in section 4 where it will be shown that a seven-mode reduced-order model is able to fairly reproduce the dynamics of the large-scale flow reorientations.

Figure 5 shows the $3 \mathrm{D}$ thermal and flow patterns of the first seven POD modes. Positive iso-surfaces of the $Q$-criterion $\left(Q=-0.5 \partial u_{i} / \partial x_{j} \times \partial u_{j} / \partial x_{i}\right.$, Hunt et al. (1988)) are used to highlight the rotating zones. The $Q$-criterion compares strain and vorticity and a positive value means that vorticity is dominant. Temperature and velocity fields of modes 4 and 7 are also displayed in figure 6 in a $2 \mathrm{D}$ vertical plane near the sidewall $x=0$. 


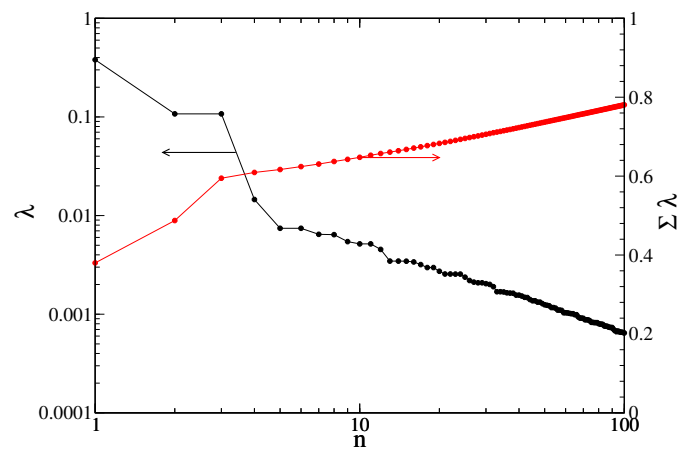

Figure 4. POD eigenspectrum normalized such that $\sum_{i} \lambda_{i}=1$ (black, left axis) and associated cumulated spectrum (red, right axis).

In addition, the angular momentum with respect to the $x$ and $y$ directions together with the symmetry properties of the first seven modes are given in table 1.

The first mode corresponds to the mean flow. The velocity field is made of two counterrotating torus-like structures confined respectively to the upper half and the lower half of the cube (the vertical component of the velocity is zero at the horizontal mid-height plane). In the upper (respectively lower) torus, the fluid flows down (up) along the adiabatic walls and turns back to the centre. The temperature field is vertically stratified from the bottom hot wall to the top cold wall. The vertical temperature gradient (heat flux) at the isothermal walls is the highest at the centre and the lowest at the corners. The velocity and temperature fields of this first mode satisfy all the symmetries of the problem and thus the associated global angular momentum is null.

Modes 2 and 3, which have the same eigenvalue, are made of a single roll around the $x$-axis and the $y$-axis respectively, which corresponds to LSC lying in $x$-planes and $y$-planes respectively. They of course possess a strong global angular momentum with respect to the $x$-axis (mode 2 ) or the $y$-axis (mode 3 ) and it can be inferred at this stage that the two modes have to be combined to form the quasi-stable diagonal rolls observed in the simulations. The two modes are identical after a rotation of $\pi / 2$ and both break the diagonal symmetry. It can be seen in figure 5 that the temperature field is correlated with the velocity field: the temperature is positive (hot) when the flow is ascending and negative (cold) when the flow is descending. Because these two modes form a pair of degenerated eigenvectors, they are not uniquely defined and they can be replaced in the POD basis by any linear combination of the two original vectors $\left(\phi_{2}, \phi_{3}\right)$ as long as the orthonormality is preserved. Namely, an infinity of eigenvector pairs $\left(\phi_{2}^{\prime}, \phi_{3}^{\prime}\right)$ can be generated, providing that they satisfy $\phi_{2}^{\prime}=\alpha \phi_{2}+\beta \phi_{3}$ and $\phi_{3}^{\prime}=\mp \beta \phi_{2} \pm \alpha \phi_{3}, \alpha$ and $\beta$ being real constants such that $\alpha^{2}+\beta^{2}=1$. For instance, we could transform the two eigenmodes of figure 5 using $\alpha=\sqrt{2} / 2$ in order to obtain two single roll-modes each lying in one of the two diagonal planes. A diagonal quasi-stable LSC would there be obtained by the activation of one mode and the cancellation of the other. However, we will preserve modes 2 and 3 as pure $x$-roll and pure $y$-roll in this study.

Mode 4 is made of 8 counter-rotating rolls along the $x$ and $y$ axis with 4 rolls in the upper half of the cube and 4 rolls in the lower half of the cube. Like the mean flow, the vertical component of the velocity is zero at the horizontal mid-height plane (see figure 6 ). The rolls transport fluid from one corner to the other, they are the largest near the adiabatic walls and shrink as they progress towards the mid $z$-axis. Combined with mode 1 (mean flow), mode 4 reinforces the corner flows within one of the two diagonal planes and softens the corner flows within the other orthogonal diagonal plane. We will 


\begin{tabular}{ccccccc} 
mode & $L_{x}$ & $L_{y}$ & \multicolumn{5}{c}{ Symmetries } \\
1 & - & - & $S_{x}$ & $S_{y}$ & $S_{z}$ & $S_{d}$ \\
2 & 0.299 & - & $S_{x}$ & $A S_{y}$ & $A S_{z}$ & - \\
3 & - & 0.299 & $A S_{x}$ & $S_{y}$ & $A S_{z}$ & - \\
4 & - & - & $A S_{x}$ & $A S_{y}$ & $S_{z}$ & $S_{d}$ \\
5 & 0.026 & - & $S_{x}$ & $A S_{y}$ & $A S_{z}$ & - \\
6 & - & 0.026 & $A S_{x}$ & $S_{y}$ & $A S_{z}$ & - \\
7 & - & - & $S_{x}$ & $S_{y}$ & $S_{z}$ & $A S_{d}$
\end{tabular}

TABLE 1. Angular momentum and symmetry properties of the first seven modes. $S_{x / y / z / d}$ and $A S_{x / y / z / d}$ denote respectively a symmetry and an anti-symmetry with respect to the planes $x=0.5, y=0.5, z=0.5$ and $x=y$.

see later that this mode stabilizes the diagonal flow states. It is interesting to note that the first four POD modes are similar to the four primary Koopman eigenfunctions identified by Giannakis et al. (2018). However, the Koopman eigenfunctions presented in their study were not symmetrised. In addition, the Koopman eigenfunctions corresponding to POD modes 2 and 3 were made of single LSC-roll located in the diagonal plane instead of the mid $x$-plane and the mid $y$-plane in our study.

Modes 5 and 6 form another pair of degenerated modes and have been chosen consistently with the pair of modes 2 and 3. Mode 5 is made of two longitudinal co-rotating rolls lying in the mid $x$-plane, one in the upper half of the cell, one in the lower half of the cell. The two rolls are connected by vertical streams along the planes $x=0$ and $x=1$. The interaction between the horizontal streams in the mid plane and the vertical streams near the walls gives rise to two small rotating structures that are responsible for a weak global angular momentum along the $x$-axis. Mode 5 possesses the same symmetries as mode 2 suggesting a strong interaction between the two modes. Mode 5 slows down or speeds up mode 2 depending on whether they are combined with same sign or opposite sign. Mode 6 is the image of mode 5 after a rotation of $\pi / 2$ and possesses the same symmetry as mode 3 . The relationship between modes 3 and 6 is thus identical to the relationship between modes 2 and 5 . Modes 5 and 6 will be referred to as boundary-layer modes in the remainder of the paper.

Mode 7 is similar to mode 4 , as it contains 8 counter-rotating structures, 4 confined in the upper half of the cell and 4 confined in the lower half of the cell. However, the rotation-axis of these rolls are aligned along diagonal axis $x=1-y$ and $x=y$ instead of the $x$-axis and the $y$-axis. These rolls bring fluid from the mid $x$-plane to the mid $y$-plane (and vice versa) and we can foresee important coupling with $x$-plane modal structures (modes 2 and 5) and $y$-plane modal structures (modes 3 and 6). We can also infer that mode 7 would destabilize a diagonal LSC. Its symmetry properties are opposite to mode 4, except for the $S_{z}$ symmetry satisfied by both modes.

To conclude, these 3D POD modes of the cubical cell can be compared with the 2D POD modes of the square cell (Podvin \& Sergent 2017). In that perspective, mode 1 would correspond to the 2D quadrupolar mode (mean flow). Modes 2 and 3 would correspond to the 2D LSC mode, the degeneracy coming from the third dimension. Modes 5 and 6 (degenerate) would correspond to the $2 \mathrm{D} L_{*}$ mode that connects the core region with the horizontal boundary layers. This last mode has been identified as a precursor for 2D reversals of the LSC. However, it is worth noting that modes 4 and 7 are pure 3D modes that have no equivalent structures in the $2 \mathrm{D}$ square case. 

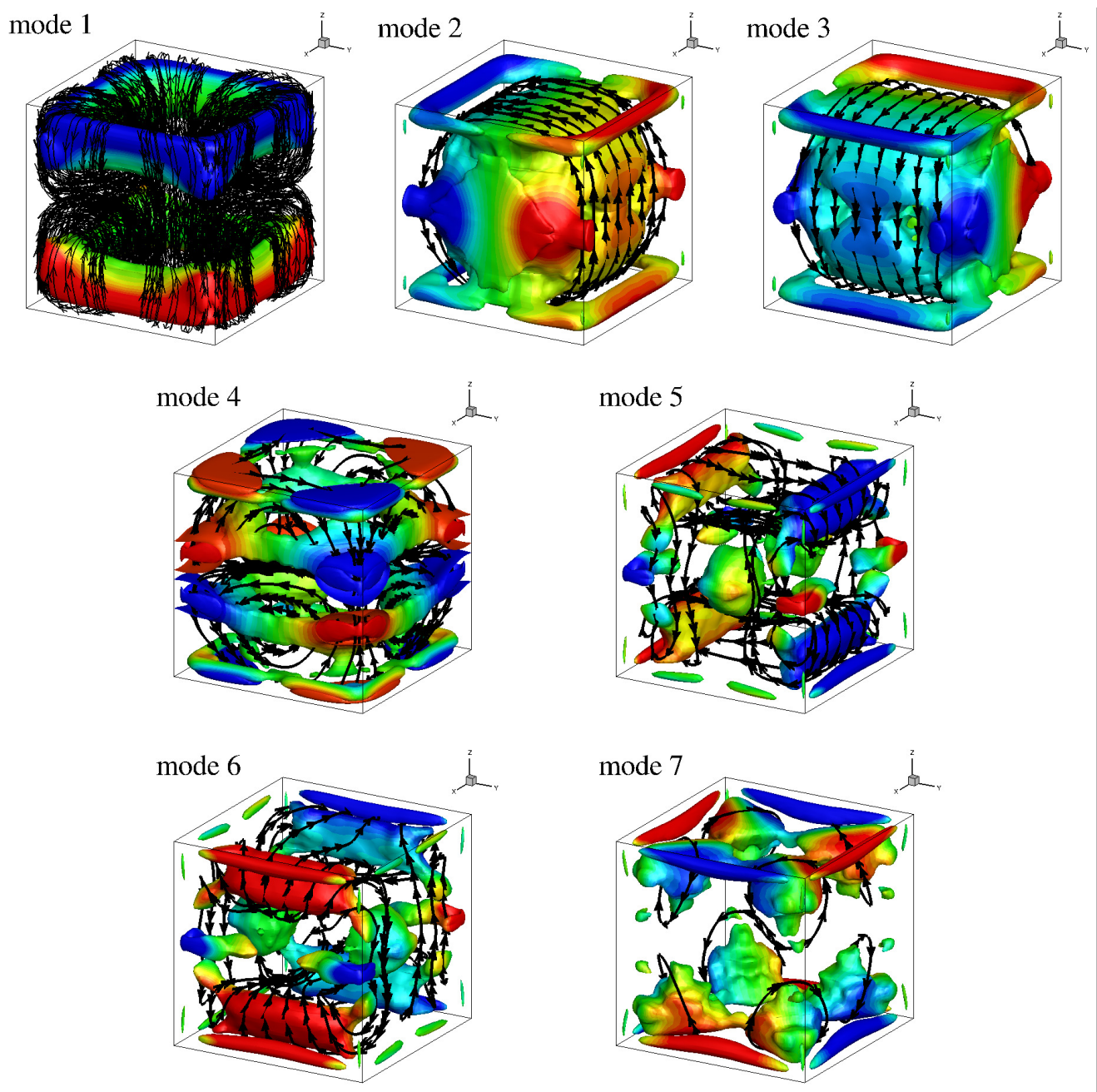

Figure 5. Seven first POD modes. Streamlines and iso-surfaces of $Q$-criterion $(Q=5$ for modes 1-3 and $Q=30$ for modes 4-7) coloured by mode temperature. Colourmap for mode temperature ranges from -0.5 (blue) to 0.5 (red).

\subsection{Temporal dynamics}

The initial (non-symmetrised) snapshot set has been projected onto the reduced POD basis of 7 modes in order to compute the associated DNS POD coefficients at discrete times $t_{i}$

$$
a_{n}\left(t_{i}\right)=\int_{\Omega} \boldsymbol{U}\left(\boldsymbol{r}, t_{i}\right) \cdot \boldsymbol{\phi}_{n}(\boldsymbol{r}) d \boldsymbol{r}
$$

The resulting time evolution of the POD coefficients is shown in figure 7 where dashed lines indicate reorientation events.

As expected, the coefficient associated with the first mode (mean flow) is nearly constant and oscillates around a mean value of $a_{1}^{e q}=\sqrt{\lambda_{1}}=0.1271$. Because modes 2 and 3 are pure $x$-roll and $y$-roll, their associated POD coefficients are correlated with the time evolution of the $x$ and $y$ components of the global angular momentum shown in figure 2. Therefore, the coefficients of modes 2 and 3 oscillate around a mean value 

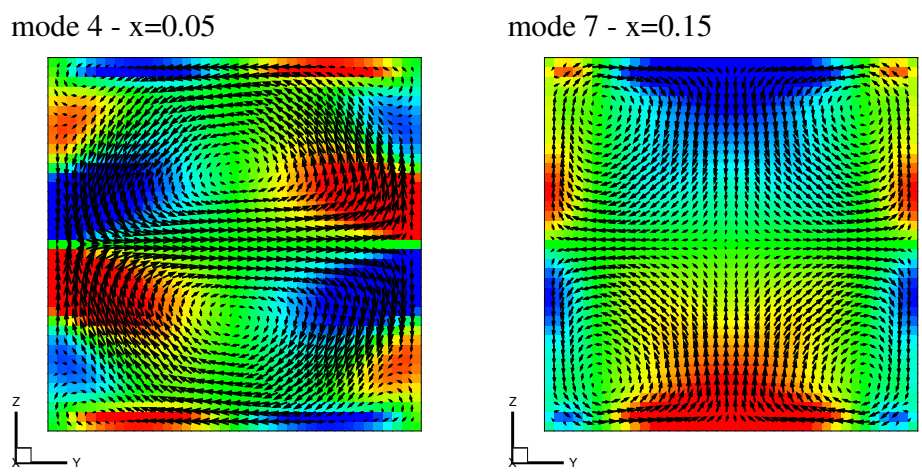

FIgURE 6. Temperature (colour) and velocity (vectors) fields in the vertical plane $x=0.05$ of POD mode 4 (left) and $x=0.15$ of POD mode 7 (right). Colourmap for temperature ranges from -0.5 (blue) to 0.5 (red).

of $a_{2 / 3}^{e q}= \pm \sqrt{\lambda_{2 / 3}}= \pm 0.0675$ during quasi-stable state period, which confirms that diagonal LSC flows are obtained by superimposing a $x$-plane LSC (mode 2) and a $y$ plane LSC (mode 3 ). We have shown in section 2.3 that reorientations occur when either the $x$-component or the $y$-component of the angular momentum changes sign. From a POD point of view, a reorientation occurs when either the coefficient $a_{2}$ or $a_{3}$ changes sign which corresponds to a reversal (a change of the sense of rotation) of either the $x$-roll (mode 2 ) or the $y$-roll (mode 3 ). This interpretation agrees with the one proposed by Vasiliev et al. (2018). We note that a cancellation or cessation of $a_{2}$ (or $a_{3}$ ) may occasionally not result in a reorientation, as can be seen at $t=5800$ in figure 7 , where $a_{2}$ drops to values close to zero for a short period before increasing back to its initial amplitude. This physically corresponds to a temporary alignment of the large-scale circulation with the side walls of the cube followed by a return to the original diagonal state.

It is worth noting that the time evolution of the $4^{\text {th }}$ POD coefficient also follows the reorientation dynamics: $a_{4}$ oscillates around a mean value of $a_{4}^{e q}= \pm \sqrt{\lambda_{4}}= \pm 0.0249$ and experiences rapid sign switches at each reorientation. The $4^{\text {th }}$ coefficient is actually strongly correlated with the product of the $2^{\text {nd }}$ and $3^{\text {rd }}$ coefficients $\left(a_{4}(t) \propto a_{2}(t) a_{3}(t)\right)$. Mode 4 could thus be interpreted as an indicator of the diagonal plane in which the LSC takes place: $a_{4}>0$ means the LSC lies in the plane $x=1-y, a_{4}<0$ means the LSC lies in the plane $x=y$.

The time evolution of the $5^{\text {th }}$ to $7^{\text {th }}$ coefficients $\left(a_{5}\right.$ and $a_{6}$ not shown) is chaotic with a dominant high frequency around $2 \times 10^{-2}$ corresponding to the rotation frequency of the diagonal LSC (see section 2.3) and does not seem to follow any specific pattern during reorientations. However, the time evolution of coefficient products $a_{5} a_{7}$ and $a_{6} a_{7}$ in figure 7 shows an interesting feature: their sign is correlated with respectively the opposite of the $2^{\text {nd }}$ and the $3^{\text {rd }}$ mode coefficients. This suggests a strong quadratic interaction between the 2D-rolls (mode 2 or 3 ), the boundary layer modes (modes 5 or 6 ) and the diagonal corner rolls (mode 7). In addition, we will see in section 4 that modes 5 to 7 are necessary in the reduced-order model to produce flow reorientations. The correlation between modes 2 and 5 , and between modes 3 and 6 , has been further confirmed by applying a moving average over 90 time units to $a_{5}(t)$ and $a_{6}(t)$, which shows a small equilibrium contribution $a_{5 / 6}^{e q}$ during quasi-stable states, much lower than the standard 
deviation $\sqrt{\lambda_{5 / 6}}$. This equilibrium contribution switches sign at each reversal of the associated planar rolls: $\operatorname{sgn}\left(a_{5}^{e q}\right)=-\operatorname{sgn}\left(a_{2}^{e q}\right)$ and $\operatorname{sgn}\left(a_{6}^{e q}\right)=-\operatorname{sgn}\left(a_{3}^{e q}\right)$.

The analysis of the time evolution of POD coefficients showed that only the four first modes are active (have a significant mean value) during quasi-stable states. We have then performed four-mode reconstructions of the flow field in order to reproduce the reorientation that happens near $t=5590$. Figure 8 shows isotherms and streamlines of three reconstructed flow fields. The left flow field in figure 8 corresponds to the mean quasi-stable state $\left(L_{x}<0, L_{y}<0\right)$ before the reorientation and has been computed such as $\boldsymbol{U}^{*}=\sqrt{\lambda_{1}} \phi_{1}-\sqrt{\lambda_{2 / 3}}\left(\phi_{2}+\phi_{3}\right)+\sqrt{\lambda_{4}} \phi_{4}$. This reconstructed flow field agrees with the time averaged flow field of figure 3 . The four modes are necessary to reproduce all the flow patterns as modes 2 and 3 capture the diagonal LSC while modes 1 and 4 capture the corner flows. The flow field shown in the middle of figure 8 corresponds to a transient state, when the $x$-roll vanishes and the LSC purely lies in a $y$-plane. It has been computed such that $\boldsymbol{U}^{*}=\sqrt{\lambda_{1}} \phi_{1}-\sqrt{\lambda_{2 / 3}} \phi_{3}$. The combination of the $y$-roll (mode 3 ) with the mean flow (mode 1 ) breaks the $S_{z}$ symmetry and forms two counterrotating structures aligned along the top front horizontal edge and the bottom back horizontal edge. This transient state is always observed during a reorientation and is in really good agreement with the transient state reported by Foroozani et al. (2017). Finally, the right flow field corresponds to the mean quasi-stable state $\left(L_{x}>0, L_{y}<0\right)$ after the reorientation, that is after the reversal of the $x$-roll, and has been computed such as $\boldsymbol{U}^{*}=\sqrt{\lambda_{1}} \phi_{1}+\sqrt{\lambda_{2 / 3}}\left(\phi_{2}-\phi_{3}\right)-\sqrt{\lambda_{4}} \phi_{4}$. Owing to the symmetry of the modes, the right flow field is the exact image of the left flow field after a $\pi / 2$ rotation.

\section{Reduced order modelling}

\subsection{Model derivation}

Using the decomposition (3.2) in Eqs (2.1-2.3) and applying Galerkin projection leads to a set of coupled ordinary differential equations of the form

$$
\frac{d a_{n}(t)}{d t}=L_{n m} a_{m}(t)+Q_{n m p} a_{m}(t) a_{p}(t)+T_{n}(t)
$$

where $T_{n}(t)$ is a closure term for capturing the effects of the truncation. The linear term can be split according to $L_{n m}=L_{n m}^{B}+L_{n m}^{D}$ where superscripts $B$ and $D$ stand for buoyancy contribution and diffusion contribution. The coefficients $L_{n m}^{B}, L_{n m}^{D}$ and $Q_{n m p}$ are defined by

$$
\begin{gathered}
L_{n m}^{B}=\int \operatorname{Pr} \phi_{\theta}^{m} \phi_{3}^{n} d \boldsymbol{r} \\
L_{n m}^{D}=\int\left[\frac{\operatorname{Pr}}{\sqrt{R a}} \frac{\partial^{2} \phi_{i}^{m}}{\partial x_{j} \partial x_{j}} \phi_{i}^{n}+\frac{\gamma^{2}}{\sqrt{R a}} \frac{\partial^{2} \phi_{\theta}^{m}}{\partial x_{j} \partial x_{j}} \phi_{\theta}^{n}\right] d \boldsymbol{r} \\
Q_{n m p}=\int\left[-\phi_{j}^{p} \frac{\partial \phi_{i}^{m}}{\partial x_{j}} \phi_{i}^{n}-\gamma^{2} \phi_{j}^{p} \frac{\partial \phi_{\theta}^{m}}{\partial x_{j}} \phi_{\theta}^{n}\right] d \boldsymbol{r} .
\end{gathered}
$$

Note that the procedure is independent of the existence of symmetry in the problem. In the present case, the model expansion has been actually restricted to the first seven modes because the $8^{\text {th }}$ mode does not possess any significant interaction with other modes (namely $Q_{8 m p} \simeq 0, \forall m<8$ and $\forall p<8$ ). The values obtained for coefficients $L^{B}, L^{D}$ and $Q$ for the first seven modes have been uploaded as supplementary material of the paper. We note that the dimension of the dynamical system is actually six, as the first mode $a_{1}$ was taken to be constant, based on its evolution observed in the DNS. 

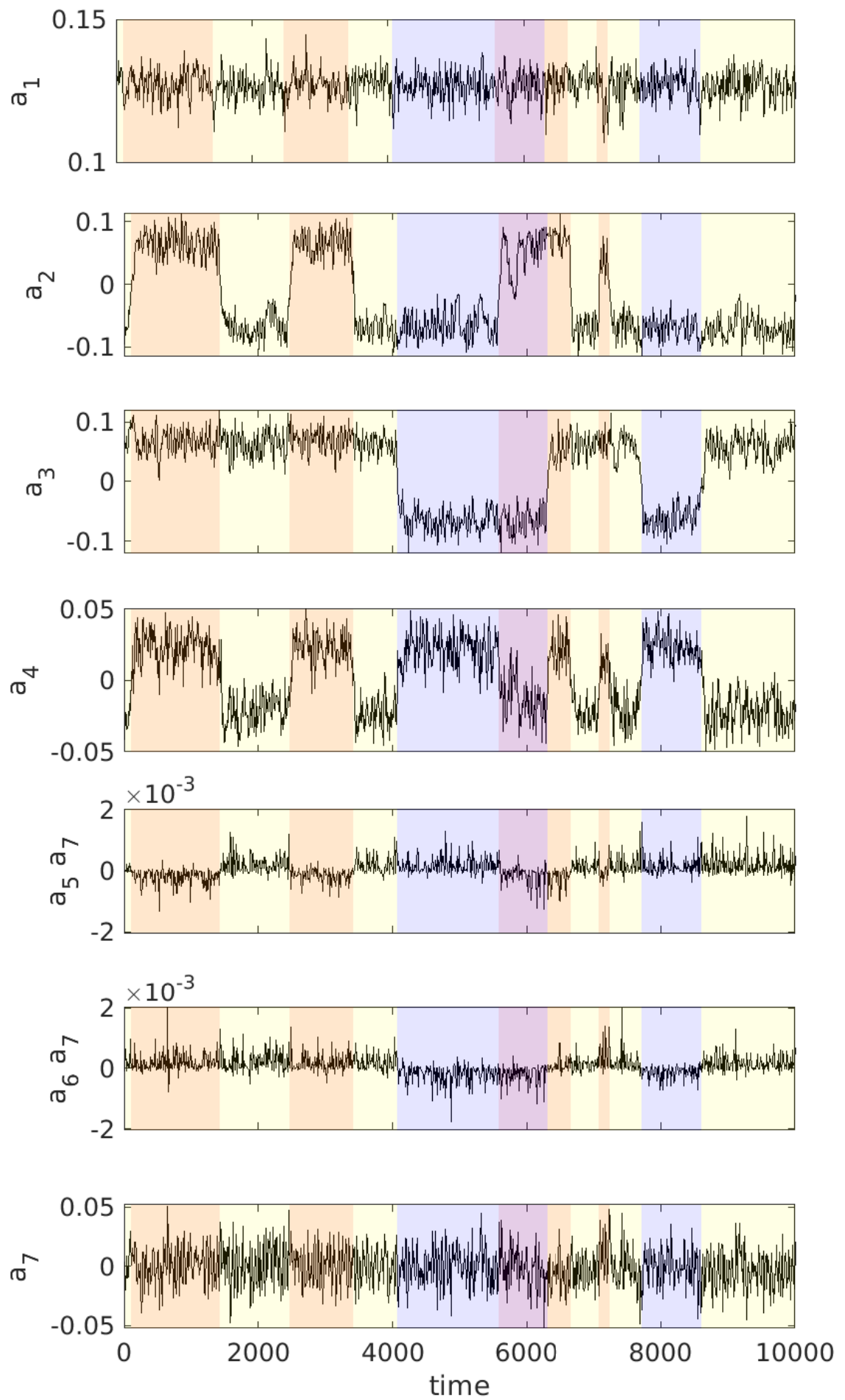

Figure 7. Time evolution of the DNS POD coefficients. Each quasi-stable state is depicted by a different colour: orange $L_{x}>0, L_{y}>0$; yellow $L_{x}<0, L_{y}>0$; blue $L_{x}<0, L_{y}<0$; purple $L_{x}>0, L_{y}<0$. 

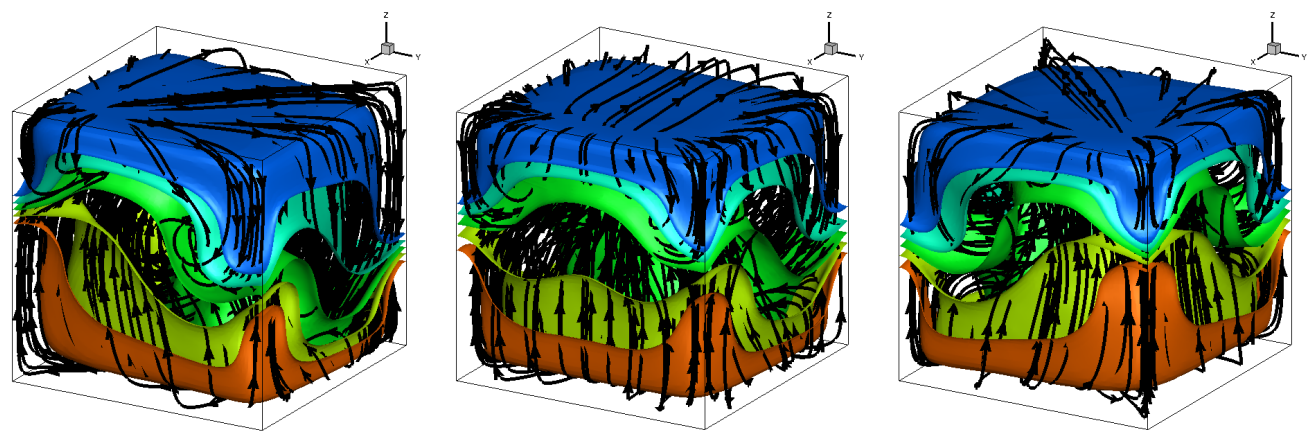

FiguRE 8. Streamlines and isotherms $\theta=\{0 ; \pm 0.05 ; \pm 0.1\}$ of reconstructed flow fields using the first four POD modes. Left: mean stable state $\left(L_{x}<0, L_{y}<0\right)$; middle: transient flow state (pure $y$-roll) and right: new mean stable state $\left(L_{x}>0, L_{y}<0\right)$.

Following Podvin \& Sergent (2015), the closure term is modelled as an equivalent dissipation term, expressed as a combination of linear and cubic terms such that

$$
T_{n}(t)=\left(L_{n m}^{A}-\alpha_{n m} \sum_{p \geqslant 2}\left|a_{p}(t)\right|^{2}\right) a_{m}(t),
$$

where $L_{n m}^{A}$ and $\alpha_{n m}$ are constants to be determined. The model has then the following form

$$
\begin{aligned}
\frac{d a_{1}}{d t}= & 0 \\
\frac{d a_{2}}{d t}= & \left(L_{22}^{T}-\alpha_{22} r^{2}\right) a_{2}+\left(L_{25}^{T}-\alpha_{25} r^{2}\right) a_{5}+0.29 a_{1} a_{2}-0.53 a_{3} a_{4} \\
& +0.33 a_{2} a_{7}+0.18 a_{5} a_{7}+0.01 a_{1} a_{5}+0.005 a_{4} a_{6} \\
\frac{d a_{3}}{d t}= & \left(L_{33}^{T}-\alpha_{33} r^{2}\right) a_{3}+\left(L_{36}^{T}-\alpha_{36} r^{2}\right) a_{6}+0.29 a_{1} a_{3}-0.53 a_{2} a_{4} \\
& -0.33 a_{3} a_{7}-0.18 a_{5} a_{7}+0.01 a_{1} a_{6}+0.005 a_{4} a_{5} \\
\frac{d a_{4}}{d t}= & \left(L_{44}^{T}-\alpha_{44} r^{2}\right) a_{4}+0.48 a_{1} a_{4}+1.06 a_{2} a_{3}+1.98 a_{2} a_{6}+1.98 a_{3} a_{5}+1.1 a_{5} a_{6}( \\
\frac{d a_{5}}{d t}= & \left(L_{55}^{T}-\alpha_{55} r^{2}\right) a_{5}+\left(L_{52}^{T}-\alpha_{52} r^{2}\right) a_{2}+0.11 a_{1} a_{2}-1.98 a_{3} a_{4} \\
& +0.4 a_{1} a_{5}-0.55 a_{4} a_{6}+0.2 a_{5} a_{7}+0.09 a_{2} a_{7} \\
\frac{d a_{6}}{d t}= & \left(L_{66}^{T}-\alpha_{66} r^{2}\right) a_{6}+\left(L_{63}^{T}-\alpha_{63} r^{2}\right) a_{3}+0.11 a_{1} a_{3}-1.98 a_{2} a_{4} \\
& +0.4 a_{1} a_{6}-0.55 a_{4} a_{5}-0.2 a_{6} a_{7}-0.09 a_{3} a_{7} \\
\frac{d a_{7}}{d t}= & \left(L_{77}^{T}-\alpha_{77} r^{2}\right) a_{7}+0.58 a_{1} a_{7} \\
& +0.28\left(a_{3} a_{6}-a_{2} a_{5}\right)+0.67\left(a_{3}^{2}-a_{2}^{2}\right)+0.41\left(a_{6}^{2}-a_{5}^{2}\right)
\end{aligned}
$$

where $r^{2}=\sum_{n=2}^{7}\left|a_{n}(t)\right|^{2}$, and $L^{T}=L^{B}+L^{D}+L^{A}$. The model is invariant by a transformation of $a_{4} \rightarrow-a_{4}, a_{2} a_{3} \rightarrow-a_{2} a_{3}, a_{5} a_{6} \rightarrow-a_{5} a_{6}$, which corresponds physically to diagonal changes. This ensures that the model dynamics are the same for all four meta-stable states.

The interaction coefficients reproduce the symmetries of the modes that were previously discussed. In particular, modes 2 and 5 (resp. 3 and 6), which have the same symmetries, are linearly coupled. The interaction coefficients of modes 1 and 4 with 
modes 2 and 5 are the same as with modes 3 and 6 . In contrast, the action of mode 7 on modes 2 and 5 is opposite from that on modes 3 and 6 . The evolution of mode 7 appears driven by dissymmetries between $a_{2}$ and $a_{3}$ as well as between $a_{5}$ and $a_{6}$, which both measure momentum and temperature differences between the $x$ and the $y$ directions. Mode 7 therefore appears as a suitable candidate for symmetry-breaking and is expected to play a significant role in the reorientations.

To determine the values of $L^{T}$ and $\alpha$, we require that the diagonal states be fixed points of the dynamical system $a_{i}^{e q}$. Examination of the DNS shown in figure 7 suggests that $a_{1}^{e q}=\sqrt{\lambda_{1}}, a_{2}^{e q}= \pm \sqrt{\lambda_{2}}, a_{3}^{e q}= \pm \sqrt{\lambda_{3}}, a_{4}^{e q}=\operatorname{sgn}\left(a_{2}^{e q} a_{3}^{e q}\right) \sqrt{\lambda_{4}}, a_{5}^{e q}=-\operatorname{sgn}\left(a_{2}^{e q}\right) \epsilon \sqrt{\lambda_{5}}$, $a_{6}^{e q}=-\operatorname{sgn}\left(a_{3}^{e q}\right) \epsilon \sqrt{\lambda_{6}}, a_{7}^{e q}=0$, where $\epsilon=0.2$. This value of $\epsilon$ was obtained by taking the conditional average of $a_{5}$ (resp. $a_{6}$ ) based on the sign of $a_{2}$ (resp. $a_{3}$ ) and averaging over about 90 dimensionless time units (corresponding to a duration of about two recirculation times $T_{c}$ and also to a minor bound for the duration of a reorientation event). A negative correlation between $a_{2}$ and $a_{5}$ (resp. $a_{3}$ and $a_{6}$ ) corresponds to a more efficient wind and a strengthened connection between the boundary layers and the core at equilibrium, which was also observed in Podvin \& Sergent (2017).

At equilibrium the linear and cubic terms balance the quadratic contribution:

$$
\left(L_{n m}^{T}-\alpha_{n m} \sum_{p=2}^{7}\left|a_{p}^{e q}\right|^{2}\right) a_{m}^{e q}+Q_{n m p} a_{m}^{e q} a_{p}^{e q}=0
$$

We then determine for each pair $(n, m)$ the total contribution $A_{n m}=L_{n m}^{T}-$ $\alpha_{n m} \sum_{p=2}^{7}\left|a_{p}^{e q}\right|^{2}$. This is straightforward for mode 4 as there is only one non-zero coefficient $A_{44}$ (diagonal term). For modes 2, 3, 5 and 6 , this involves setting the relative importance between diagonal and off-diagonal terms, which can be characterized by a coupling term $X_{i}$, whose definition and determination is detailed in appendix $\mathrm{A}$. In the case of mode 7 , the value of the coefficient $A_{77}$ which should balance the quadratic contribution cannot be determined in a straightforward manner, since the mode is zero at equilibrium. The linear and cubic coefficients for mode 7 were therefore adjusted empirically in order to limit the energy in that mode, which tends to be overestimated as it is the highest-order mode left in the truncation.

Once $A_{n m}$ is known, it remains to determine the respective values of $L_{n m}^{T}$ and $\alpha_{n m}$. As was shown in Podvin \& Sergent (2015), the additional terms are modelled as

$$
L^{A}-\alpha r^{2} \sim-C\langle k\rangle^{1 / 2} \frac{1}{2}\left(1+\frac{k}{\langle k\rangle}\right),
$$

where $k=r^{2}$ is the energy of the fluctuating modes in the truncation and $\langle k\rangle$ is its temporal average $\left(\langle k\rangle \simeq r_{e q}^{2}=\sum_{p=2}^{7}\left|a_{p}^{e q}\right|^{2}\right)$. If one identifies term by term the expressions for each mode, one finds that

$$
L_{n m}^{A}=-\alpha_{n m}\langle k\rangle .
$$

Since $A_{n m}=L_{n m}^{B}+L_{n m}^{D}+L_{n m}^{A}-\alpha_{n m} \sum_{p=2}^{7}\left|a_{p}^{e q}\right|^{2}$, one obtains

$$
\alpha_{n m}=-\frac{A_{n m}-L_{n m}^{B}-L_{n m}^{D}}{2 \sum_{p=2}^{7}\left|a_{p}^{e q}\right|^{2}} .
$$

However, we have noticed that the behaviour of the model did not appear to depend strongly on the choice of $\alpha$. 


$\begin{array}{cccccccc} & \text { Mode i } & 2 & 3 & 4 & 5 & 6 & 7 \\ \text { DNS } & \sqrt{\lambda_{i}} & 0.0675 & 0.0675 & 0.0249 & 0.0178 & 0.0178 & 0.0166 \\ & T_{i} \text { (filtered) } & 884 & 1514 & 703 & & & \\ \text { Reference model } & \left\langle a_{i}^{2}\right\rangle^{1 / 2} & 0.058 & 0.059 & 0.018 & 0.012 & 0.012 & 0.041 \\ & T_{i} \text { (filtered) } & 1470 & 1433 & 815 & & & \end{array}$

TABLE 2. Statistics of the modes integrated in the model over a period of 50,000 time units with noise (noise level 0.0012). $T_{i}$ is defined as the mean interval between zeros of $a_{i}^{f}$ where $a_{i}^{f}$ is the filtered time series of $a_{i}$ obtained by moving average over two recirculation times (90 units). Intervals smaller than 90 units are excluded from the statistics.

\subsection{Model integration}

\subsubsection{Histories}

The values of the different parameters $\alpha_{n m}$ and $L_{n m}^{T}$ used in the model integration have been uploaded as supplementary material of the paper. We checked that the behaviour of the dynamical system was robust in a neighbourhood of the parameters chosen. The model was integrated over a period of 50,000 time units from a random initial condition close to one of the equilibria and compared with the DNS. A random noise perturbation of amplitude $1.2 \times 10^{-3}$ was added to the equations. The determination of the noise level is discussed in appendix A. The rms of the amplitudes $a_{i}$ in the model are reported in table 2. Except for the last mode of the truncation, which is overestimated by a factor of 4 , a good agreement between the model and the DNS - within 30\% - is observed. We note that the modes retained in the seven-mode truncation represent only $63 \%$ of the total combined kinetic and thermal energy.

Histories for the model amplitudes $a_{i}$ are presented in figure 9 over a period of 10,000 time units and compared with their counterparts in the DNS shown in figure 7. A good agreement is observed in the behaviour of the modes. The system spends long periods of time near the equilibria $\left|a_{2}\right|=\left|a_{2}^{e q}\right|,\left|a_{3}\right|=\left|a_{3}^{e q}\right|$, separated by switches from one diagonal to another, which corresponds to individual sign changes of $a_{2}$ or $a_{3}$. We note that these sign changes can occasionally occur at the same time in the model (see around $t=2250$ and $t=3600$ in figure 9). Although this was not observed over the duration of the present DNS (which corresponds to a limited number of switches), simultaneous switches i.e reversals along a given diagonal were observed by Giannakis et al. (2018). However, infrequent cessations of either mode 2 or 3 which were observed in the DNS are also present in the model: $a_{3}$ cancels around $t=6100$ or $a_{2}$ cancels around $t=8500$. During these events, the LSC stabilizes in a $x$ or a $y$ plane (wall-parallel roll), even so this does not last more than 100 time units.

The coupling between the modes reported earlier in the DNS appears to be captured by the model. Firstly, as in the DNS, the amplitude of mode 4 is almost perfectly correlated in the model with the product $a_{2} a_{3}$ (the correlation coefficient is larger than 0.9 in both cases) and one has $a_{4} \sim C a_{2} a_{3}$ where $C \sim 5$ in the model and $C \sim 6$ in the DNS. Secondly, as in the DNS, figure 9 shows that although modes 5, 6 and 7 appear to fluctuate randomly on an individual basis, large excursions of the product $a_{5} a_{7}$ (respectively $a_{6} a_{7}$ ) tend to have the same sign as the opposite of $a_{2}$ (resp. $a_{3}$ ), which therefore indicates a connection between the interaction of these oscillating modes over long time scales and the state of the LSC. The agreement is confirmed by the comparison of phase portraits provided in figure 10. The dynamics of mode 2 and mode 5 is correctly captured by the model. As noted earlier, the amplitude of mode 7 is overestimated by the model but 

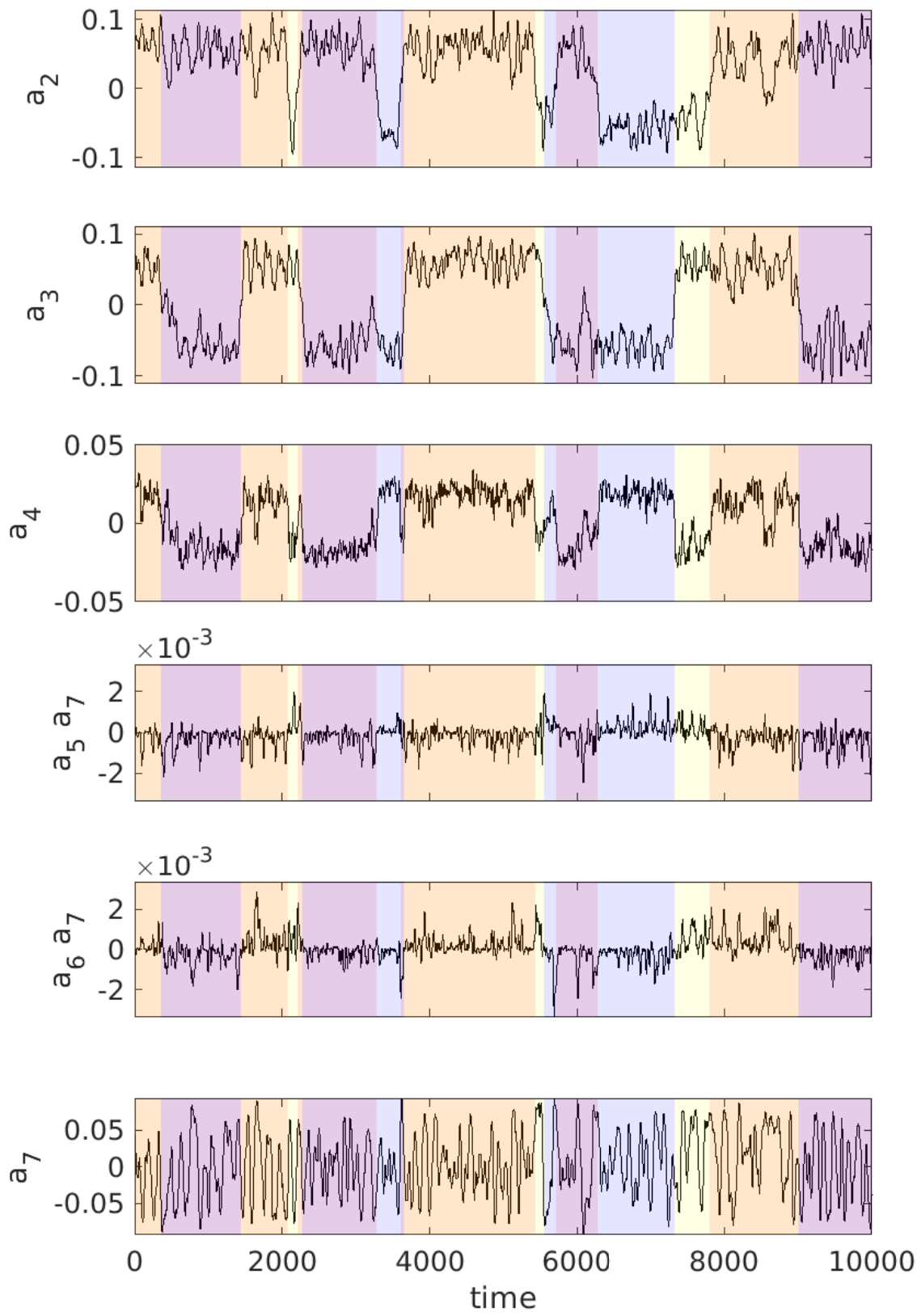

Figure 9. Time evolution of the modelled POD coefficients. Each quasi-stable state is depicted by a different colour (same colour legend as figure 7)

its qualitative behaviour appears to be correct. A 2-D histogram of modes 2 and 3 was compared with the DNS in figure 11 - we note that unlike the phase portraits, symmetries were applied to the model as well as the DNS data. The agreement is good and confirms that the system spends most of its time near the diagonal states in both the model and the DNS. 

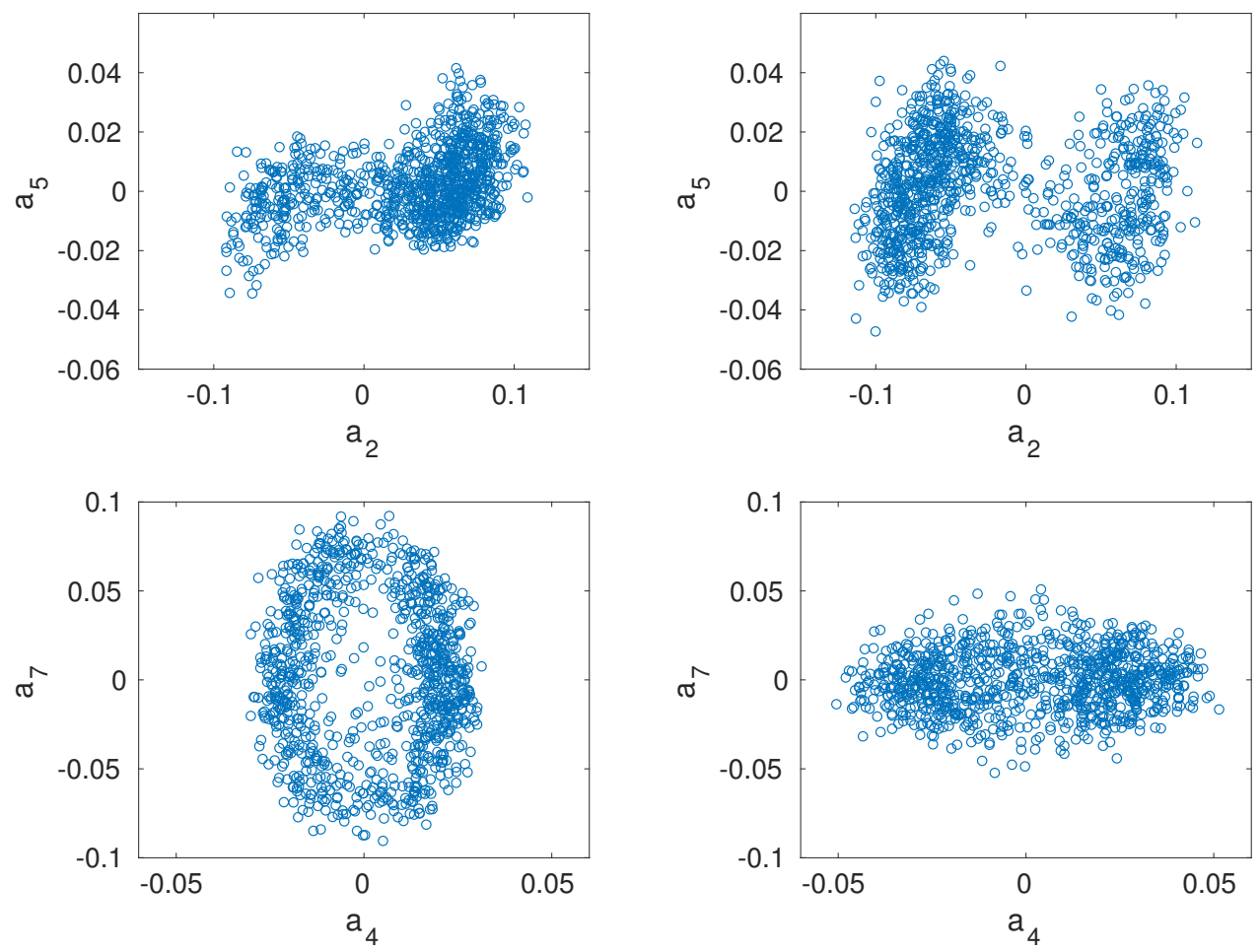

Figure 10. Phase portrait of the amplitudes in the model (left) and in the DNS (right) top row: $a_{2}$ and $a_{5}$ bottom row: $a_{4}$ and $a_{7}$
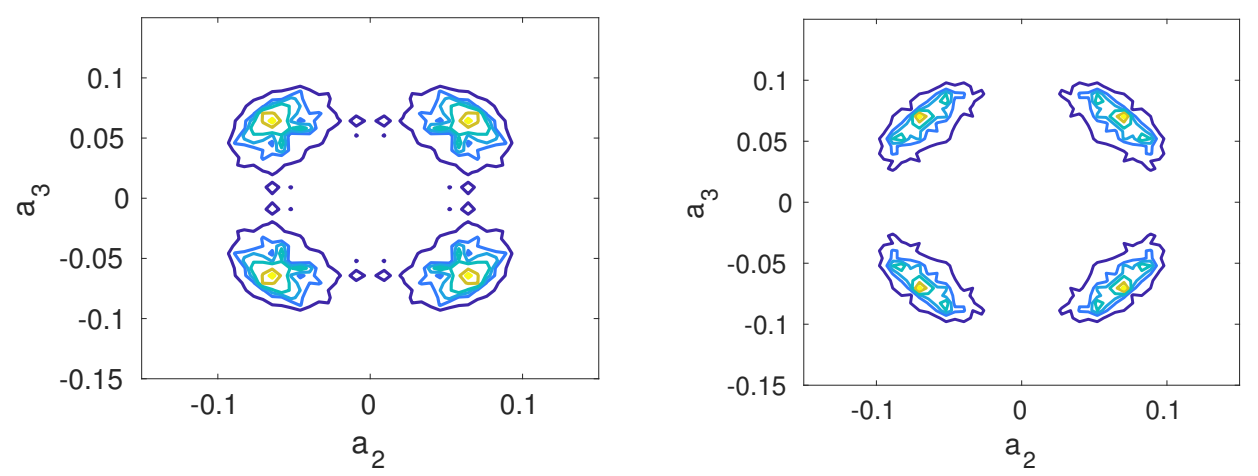

FIGURE 11. Histogram in the $\left(a_{2}, a_{3}\right)$ space. Left: model with noise amplitude $1.2 \times 10^{-3}$, right: DNS. Both DNS and model data are symmetrized. Isocontours $\{0.2 ; 0.3 ; 0.4 ; 0.6 ; 0.8 ; 1\}$.

\subsubsection{Time scales}

To characterize the times between the switches, we measure the times $T_{i}, i \in\{2,3,4\}$ separating zeros of $a_{i}$. Due to strong oscillations in the coefficients which could create numerical artefacts, we used a filtered version of the time series where a moving average over $T=90$ dimensionless time units was applied to $a_{i}$. As mentioned earlier, this time scale represents a lower bound for the time required for a full diagonal switch and corresponds to two recirculation times. Values smaller than this time scale were therefore also excluded from the statistics. Results are given in table 2 and show that the model 
displays time scales between switches of the order of several hundred units, in good agreement with the DNS. We note that statistics for the DNS were obtained over a shorter period (10,000 time units) than the model, which was integrated over a longer period of time (50,000 time units). This accounts for the larger discrepancy between $T_{2}$ and $T_{3}$ observed in the DNS compared to the model.

Temporal spectra were computed for both the DNS and the model POD amplitudes. We checked that the time scales observed were independent of the time scale chosen for the noise signal in the model (10 dimensionless time units). Both sets were sampled at a resolution of 10 dimensionless time units. Results are shown in figure 12 for selected modes 2 and 7. Due to the long period between the switches relative to the total integration time, the characteristic time scale of switches is not clearly apparent in the spectra. A short time scale of 45 units can be identified in the DNS, versus a characteristic scale of about 110 in the model. This is larger by a factor of 2 than the recirculation time $T_{c}$, which was identified in the DNS, but much less than the characteristic period between reorientations. Given the size of the truncation which captures only about $60 \%$ of the combined energy, we can consider that the agreement is relatively satisfactory. The model is therefore able to exhibit both slow and fast dynamics on time scales comparable to those observed in the DNS.

Below the short time scale, one can notice on the spectra that remaining frequencies have higher amplitudes in the DNS compared to the model (this is also noticeable when comparing figures 7 and 9). This is not surprising given that the smallest spatial scales associated to the smallest time scales are only accounted for in the model through the addition of noise and one cannot expect to capture these temporal scales with a low order truncation.

\subsection{Physical interpretation of the model}

\subsubsection{Four-mode model}

To understand the influence of the different modes, and in particular the higher-order ones, we consider a restriction of the model to the first four modes. The model has then the following form:

$$
\begin{aligned}
& \frac{d a_{1}}{d t}=0 \\
& \frac{d a_{2}}{d t}=\left(L_{22}^{T}-\alpha_{22} \sum_{k=2}^{4} a_{k} a_{k}\right) a_{2}+0.29 a_{1} a_{2}-0.53 a_{3} a_{4} \\
& \frac{d a_{3}}{d t}=\left(L_{33}^{T}-\alpha_{33} \sum_{k=2}^{4} a_{k} a_{k}\right) a_{3}+0.29 a_{1} a_{3}-0.53 a_{2} a_{4} \\
& \frac{d a_{4}}{d t}=\left(L_{44}^{T}-\alpha_{44} \sum_{k=2}^{4} a_{k} a_{k}\right) a_{4}+0.48 a_{4} a_{1}+1.06 a_{2} a_{3}
\end{aligned}
$$

where $L_{22}^{T}=L_{33}^{T}, \alpha_{22}=\alpha_{33}=\alpha$. As seen above, the model is characterized by four fixed points corresponding to the diagonal states $\left(a_{2}^{e q}= \pm a= \pm \sqrt{\lambda_{2}}, a_{3}^{e q}= \pm a= \pm \sqrt{\lambda_{3}}, a_{4}=\right.$ $\left.\operatorname{sgn}\left(a_{2} a_{3}\right) \sqrt{\lambda_{4}}\right)$ observed in the DNS. It also displays four fixed pure (wall-parallel) roll states $\left(a_{2 s}= \pm a_{s}, a_{3}=a_{4}=0\right)$ and $\left(a_{2}=0, a_{3}= \pm a_{s}, a_{4}=0\right)$.

A remarkable feature of the four-mode model is that it reproduces the nearly perfect correlation between $a_{4}$ and $a_{2} a_{3}$ observed in the DNS. We therefore have

$$
a_{4} \sim C a_{2} a_{3} \text { with } C>0 .
$$



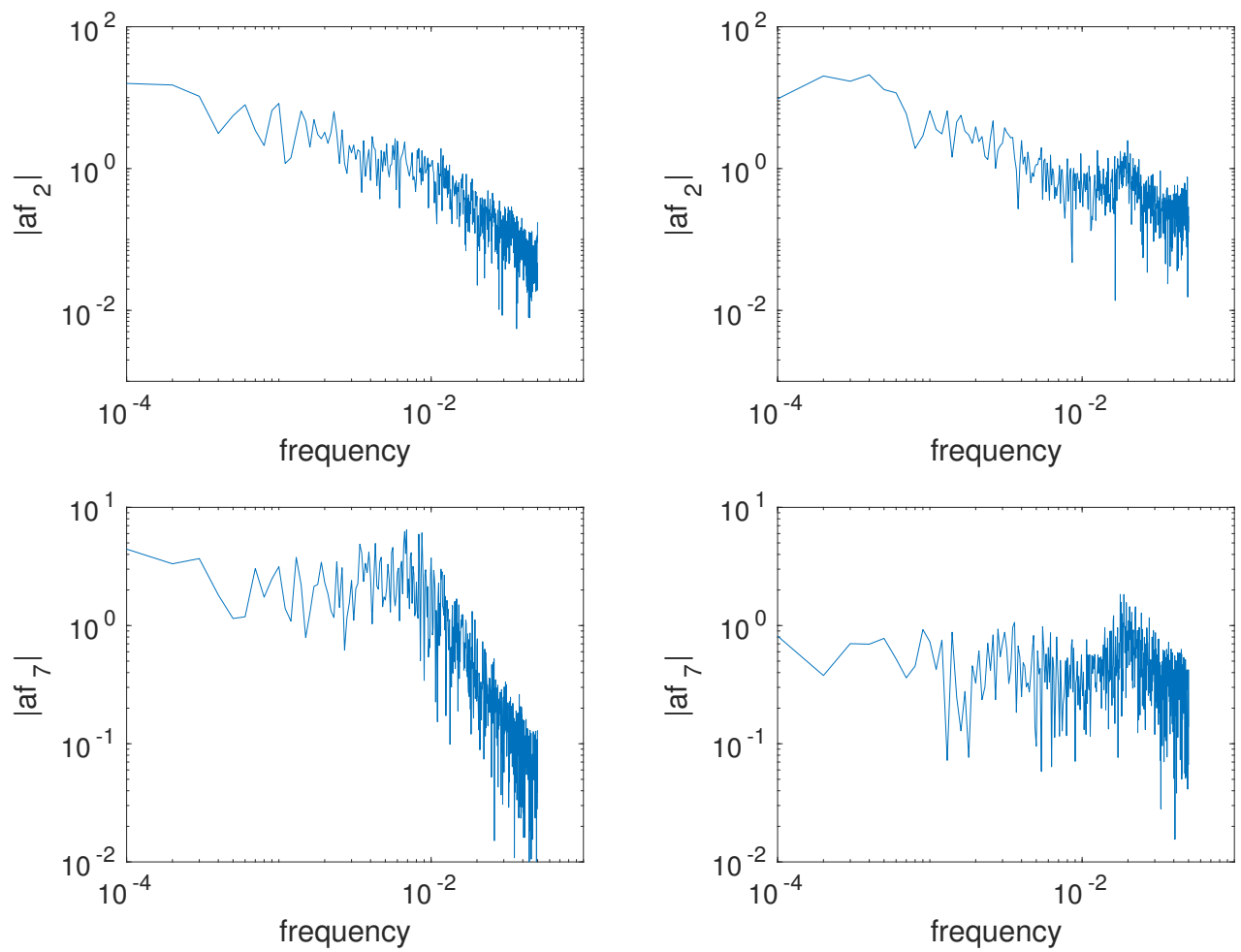

FIgURE 12. Fourier transform modulus $\left|a f_{i}\right|=\left|\hat{a}_{i}\right|$ of POD amplitudes in the model (left) and in the DNS (right). Top row: $a_{2}$; bottom row: $a_{7}$.

This means that: (i) the effect of mode 4 is analogous to a feedback cubic term and (ii) the dynamics take place on the centre manifold $a_{4}=C a_{2} a_{3}$, where the dynamical system can be written as

$$
\begin{aligned}
\frac{d a_{2}}{d t} & =\left(\gamma-\alpha a_{2}^{2}-(\alpha-\delta) a_{3}^{2}-\alpha C^{2} a_{2}^{2} a_{3}^{2}\right) a_{2} \\
\frac{d a_{3}}{d t} & =\left(\gamma-\alpha a_{3}^{2}-(\alpha-\delta) a_{2}^{2}-\alpha C^{2} a_{2}^{2} a_{3}^{2}\right) a_{3}
\end{aligned}
$$

where $\delta=-0.53 C$ and $\gamma=L_{22 / 33}^{T}+0.29 a_{1}$. Stability analysis around the equilibria can then be performed in the $\left(a_{2}, a_{3}\right)$ subspace. For the diagonal equilibria, one has $\gamma-(2 \alpha-$ $\delta) a^{2}-\alpha C^{2} a^{4}=0$, so that the Jacobian of the diagonal states $\left( \pm a, \pm a, \operatorname{sign}\left(a_{2} a_{3}\right) C a^{2}\right)$ can be expressed as:

$$
J_{d}^{2}=\left[\begin{array}{cc}
-2 \alpha\left(1+C^{2} a^{2}\right) a^{2} & -2(\alpha-\delta) a^{2}-2 \alpha C^{2} a^{4} \\
-2(\alpha-\delta) a^{2}-2 \alpha C^{2} a^{4} & -2 \alpha\left(1+C^{2} a^{2}\right) a^{2}
\end{array}\right],
$$

which has two eigenvalues $-2 \delta a^{2}$ and $2 \delta a^{2}-4 \alpha\left(1+C^{2} a^{2}\right) a^{2}$. For a roll state defined by $a_{2}= \pm a_{s}, a_{3}=a_{4}=0$, one has $\gamma-\alpha a_{s}^{2}=0$, and the associated Jacobian is:

$$
J_{s}^{2}=\left[\begin{array}{cc}
-2 \alpha a_{s}^{2} & 0 \\
0 & \delta a_{s}^{2}
\end{array}\right]
$$

An equivalent formulation is found for $\left(a_{2}=0, a_{3}=a_{s}, a_{4}=0\right)$ by switching the two rows.

The stability of the roll states and the diagonal states therefore depends on the sign 


\begin{tabular}{ccccc} 
Eigenvalues $\mu_{i}$ & $-0.16 \pm 0.18 i$ & $-0.005 \pm 0.056 i$ & -0.089 & -0.049 \\
\hline Eigenvectors & & & & \\
$\xi_{i j}, j=2$ & $0.08 \pm 0.11 i$ & $0.14 \pm 0.24 i$ & 0.059 & -0.68 \\
$\xi_{i j}, j=3$ & $0.08 \pm 0.11 i$ & $-(0.14 \pm 0.24 i)$ & -0.059 & -0.68 \\
$\xi_{i j} j=4$ & 0.69 & 0 & 0 & 0.007 \\
$\xi_{i j}, j=5$ & $0.10 \pm 0.47 i$ & $0.20 \pm 0.14 i$ & -0.68 & 0.19 \\
$\xi_{i j}, j=6$ & $0.10 \pm 0.47 i$ & $-(0.20 \pm 0.14 i)$ & 0.68 & 0.19 \\
$\xi_{i j}, j=7$ & 0 & -0.85 & -0.23 & 0
\end{tabular}

TABLE 3. Linear stability eigenvalues $\mu_{i}$ of the system around the equilibrium $\left(a 1, a_{2}^{e q}, a_{3}^{e q}, a_{4}^{e q}, a_{5}^{e q}, a_{6}^{e q}, 0\right)$ and eigenvectors $\boldsymbol{\xi}_{i}$ expressed in the basis $\left(a_{i}, 2 \leqslant i \leqslant 7\right)$.

of $\delta=-0.53 C$. For $\delta<0$, which corresponds to the Galerkin projection, the diagonal states are unstable and the roll states are stable (all eigenvalues are negative). However the DNS corresponds to the situation where the roll states are unstable and the diagonal states are stable, i.e the case $\delta>0$ (or equivalently a positive quadratic interaction between modes 2, 3 and 4). The addition of higher-order modes is therefore necessary to capture the correct dynamics.

\subsubsection{Linear stability analysis of the full model}

To investigate the influence of the modes in more detail, we study the stability of the diagonal equilibria in the full model. Table 3 shows the eigenvalues $\mu_{i}$ and eigenvectors $\underline{\xi}_{i}$ expressed in the basis of modes $a_{j}$. Eigenvectors are either symmetric or antisymmetric with respect to $a_{2}, a_{3}, a_{5}, a_{6}$ and are respectively orthogonal to $a_{7}$ or to $a_{4}$. All equilibria are stable in the model. The most stable mode $(\mu=-0.16 \pm 0.18 i)$ contains predominantly mode $a_{4}$ and has no component along $a_{7}$. In contrast, the least stable eigenmode $(\mu=$ $-0.005 \pm 0.056 i)$ has a strong component in $a_{7}$, confirming the destabilizing potential of the corner flow, in agreement with other observations (Giannakis et al. 2018). This least stable eigenvalue has an imaginary part of about 0.05 , which corresponds to the characteristic time scale of about 110 found in the dynamics of the model.

\subsubsection{Sensitivity of the model}

Another way to understand the role played by the different modes is to study the effect of changes in the parameters of the model. Table 4 compares the statistics of the POD amplitudes when small changes are applied to the model coefficients. Other parameters such as the initial condition and the noise signal used to compute the reference statistics are kept the same. The cubic terms were also kept constant when adjusting the linear terms since they were not found to affect significantly the model dynamics. Results were confirmed by linear stability analysis.

The first case in table 4 corresponds to a scenario where the intensities of modes 5 and 6 at equilibria are decreased by half, which corresponds to an equivalent decrease in the coupling term $X_{5 / 6}$ (see appendix A). Physically this means that the connection between the large-scale circulation and the boundary layer is less intense, and we would expect the equilibrium to be less stable, leading to a decrease in the inter-switch mean time. This is indeed what is observed in the model. We then examine the effect of modifying the linear terms $L^{T}$ in the equations for modes 5 and 6 , which is equivalent to adjusting the coupling terms $X_{5 / 6}$ (see appendix A). Given the energy ratio between mode 2 and mode 5 (resp. mode 3 and mode 6 ), it is clear that a moderate change in the linear term $L_{52 / 63}^{T}$ will correspond to a larger modification of the diagonal term $L_{55 / 66}^{T}$. Table 4 shows 


$\begin{array}{cccccccc} & \text { Mode i } & 2 & 3 & 4 & 5 & 6 & 7 \\ \text { Reference model } & \left\langle a_{i}^{2}\right\rangle^{1 / 2} & 0.058 & 0.059 & 0.018 & 0.012 & 0.012 & 0.041 \\ & T_{i} & 1470 & 1433 & 815 & & & \\ a_{5 / 6}^{\prime e q}=0.5 a_{5 / 6}^{e q} & \left\langle a_{i}^{2}\right\rangle^{1 / 2} & 0.058 & 0.058 & 0.018 & 0.012 & 0.012 & 0.041 \\ & T_{i} & 1177 & 1268 & 737 & & & \\ L_{55 / 66}^{\prime T}=0.75 L_{55 / 66}^{T} & \left\langle a_{i}^{2}\right\rangle^{1 / 2} & 0.056 & 0.056 & 0.017 & 0.016 & 0.017 & 0.043 \\ & T_{i} & 1092 & 1230 & 810 & & & \\ L_{55 / 66}^{\prime T}=1.25 L_{55 / 66}^{T} & \left\langle a_{i}^{2}\right\rangle^{1 / 2} & 0.061 & 0.061 & 0.018 & 0.010 & 0.010 & 0.041 \\ T_{77}^{\prime T}=0.75 L_{77}^{T} & T_{i} & 1791 & 1574 & 892 & & & \\ L_{77}^{\prime} & \left\langle a_{i}^{2}\right\rangle^{1 / 2} & 0.056 & 0.056 & 0.015 & 0.014 & 0.014 & 0.056 \\ L_{7}^{\prime T}=1.25 L_{77}^{T} & T_{i} & 751 & 698 & 420 & & & \\ & \left\langle a_{i}^{2}\right\rangle^{1 / 2} & 0.062 & 0.062 & 0.019 & 0.012 & 0.012 & 0.038\end{array}$

TABLE 4. Statistics of the integrated modes for slight variations of the model coefficients. The noise level is $1.210^{-3}$.

that for a decrease in $L_{55 / 66}^{T}$ ( $L_{55 / 66}^{T}$ is negative), the time between switches increases, the energy in modes 2 and 3 increases, while that in modes 5 and 6 decreases. The energy in mode 4 remains about constant. The trends are opposite for an increase in $L_{55 / 66}^{T}$. This supports the idea that increasing the energy transfer to the boundary layer modes 5 and 6 leads to more destabilization associated with a higher energy content in these modes. The behaviour of the model therefore highlights the importance of the boundary layer modes for the reorientation process.

Similarly, table 4 shows that a decrease in $L_{77}^{T}$ ( $L_{77}^{T}$ is negative) leads to an increase of the time between switches, and a decrease in the energy of mode 7 , along with an increase in the energy of modes 2,3 as well as mode 4 . The energy of modes 5 and 6 does not appear to change significantly. The trends are reversed for an increase in $L_{77}^{T}$. The importance of mode 7 for the destabilization process is therefore confirmed. The changes in the inter-switch periods due to modifications in $L_{77}^{T}$ are stronger than those observed for $L_{55 / 66}^{T}$.

\section{Conclusion}

Reorientations of the large-scale circulation in a turbulent Rayleigh-Bénard cubic cell were studied using direct numerical simulation for air at $R a=10^{7}$. Proper Orthogonal Decomposition was applied to the joint temperature and velocity fields in the full volume. The database was extended in order to display the statistical symmetries of the flow. The flow was characterized by four quasi-stable states, corresponding to the alignment of the large-scale circulation along one or another diagonal of the cube in a given direction. We found that each quasi-stable state consists of a superposition of four spatial modes representing three types of structures: (i) a structure carrying the time-averaged temperature and velocity fields and consisting of two stacked counter-rotating torus-like structures ; (ii) two large-scale rolls, each of which is parallel with two opposite vertical walls of the cube and carries the angular momentum of the cell in the direction orthogonal to the walls; (iii) a double layer of four rolls, each along an axis parallel to one of the side walls (each roll transports fluid from one corner to another), the effect of which is to strengthen ("focus") the circulation along one of the two diagonal plane. The first three types of structures have nearly constant amplitudes, modulated by oscillations on 
a fast time scale on the order of the recirculation time $T_{c}$ - about 45 dimensionless time units. In addition, each large-scale roll interacts with a boundary layer mode, consisting of two co-rotating rolls aligned with vertical walls. The amplitude of these modes, which control the connection between the boundary layer and the core, is dominated by strong oscillations on the time scale $T_{c}$.

The flow spends long periods of time (several hundreds of dimensionless time units) hovering near one of the quasi-stable states with occasional, fast reorientations towards another quasi-stable state. During a reorientation, the main circulation changes diagonals. The amplitude of one large-scale roll changes signs, and the side corner flows are reversed. Reorientations were found to involve the boundary layer modes, as well as a symmetrybreaking POD mode. This mode consists of eight corner roll structures, whose axis is aligned with the diagonals. It transfers the flow from one mid-plane to another adjacent, orthogonal one, effectively redirecting the angular momentum in a direction parallel to the walls. Its amplitude is also dominated by oscillations on the same fast time scale.

A POD-based model involving these modes was derived from the Navier-Stokes equations. The model reproduces the dynamical behaviour of the amplitudes of the POD modes. In particular, it captures the oscillations of the boundary layer modes as well as the long time scales of the reorientation process. Analysis of the low-dimensional system confirms the destabilizing role played by the corner rolls and suggests that the boundary layer modes play a double role. On the one hand, they help maintain the stability of the equilibrium by strengthening the connection between the large-scale circulation and the boundary layer fluctuations. On the other hand, their interaction with the corner rolls is essential for the reorientation process.

To conclude, the numerical results and associated POD model presented in this paper should be confirmed by experiments in air, though experimental evidence of flow reorientations in cubical cavities filled with water have already been reported in the literature (Bai et al. 2016; Vasiliev et al. 2016).

\section{Acknowledgements}

This work was granted access to the HPC resources of IDRIS under the allocation 2019A0042B00209 attributed by GENCI (Grand Equipement National de Calcul Intensif).

\section{Appendix A. Determining the adjustable coefficients}

In the case of modes $2,3,5$ and 6 , we need to set two adjustable contributions, the sum of which must balance the quadratic contribution. The quadratic contribution can be split into different terms:

- interactions which involve the first four modes (dominant at equilibrium);

- interactions which involve mode 5 or mode 6 but not mode 7 (symmetry-preserving interactions);

- interactions involving mode 7 (symmetry-breaking interactions), which are zero at equilibrium.

We illustrate this by considering the quadratic terms in the equation for mode 2 (or equivalently 3):

$$
\frac{d a_{2}}{d t}=\left(L_{22}^{T}-\alpha_{22} r^{2}\right) a_{2}+\left(L_{25}^{T}-\alpha_{25} r^{2}\right) a_{5}+Q_{22}+Q_{25}+Q_{27}
$$

where $Q_{22}=0.29 a_{1} a_{2}-0.53 a_{3} a_{4}, Q_{25}=0.01 a_{1} a_{6}+0.005 a_{4} a_{5}$ and $Q_{27}=0.33 a_{2} a_{7}+$ 
$0.18 a_{5} a_{7}$. At equilibrium, we need to have

$$
0=A_{22} a_{2}^{e q}+A_{25} a_{5}^{e q}+Q_{22}^{e q}+Q_{25}^{e q},
$$

where we have $A=L^{D}+L^{B}+L^{A}-\alpha r_{e q}^{2}$. Let

$$
X_{2}=A_{22} a_{2}^{e q}+Q_{22}^{e q}=-A_{25} a_{5}^{e q}-Q_{25}^{e q} .
$$

The question is to determine $X_{2}$ (or equivalently the values of $A_{22}$ and $A_{25}$ ). It can be seen from equation (A 3 ) that $A_{22}$ and $A_{25}$ cannot be chosen independently, as each of them depends on the value of $X_{2}$. For consistency of the truncation we require that modes 2 and 3 are balanced independently of the higher-order modes, which means that $X_{2}=X_{3}=0$.

For mode 5 (or equivalently 6 ), the evolution equation reads

$$
\frac{d a_{5}}{d t}=\left(L_{55}^{T}-\alpha_{55} r^{2}\right) a_{5}+\left(L_{52}^{T}-\alpha_{52} r^{2}\right) a_{2}+Q_{25}+Q_{55}+Q_{57}
$$

where $Q_{52}=0.11 a_{1} a_{2}-1.98 a_{3} a_{4}, Q_{55}=0.4 a_{1} a_{5}-0.55 a_{4} a_{6}, Q_{57}=0.2 a_{6} a_{7}+0.09 a_{2} a_{7}$. At equilibrium

$$
0=A_{52} a_{2}^{e q}+A_{55} a_{5}^{e q}+Q_{52}^{e q}+Q_{55}^{e q},
$$

which leads to the definition of a coupling term $X_{5}$ such that

$$
X_{5}=A_{55} a_{5}^{e q}+Q_{55}^{e q}=-A_{52} a_{2}^{e q}-Q_{22}^{e q} .
$$

To determine $X_{5}$, we chose to make the total contribution from the linear terms $\left|A_{52} a_{2}\right|$ and $\left|A_{55} a_{5}\right|$ approximately equal. The parameters given in the supplementary material correspond to $X_{5}=X_{6}=3.8 \times 10^{-4}$. Since there is some arbitrariness in this choice, we checked that the model behaviour was robust in a neighbourhood of the parameters chosen.

A last adjustable parameter to be determined is the noise amplitude. The noise is supposed to represent the high-frequency component of the unresolved stress tensor which is not captured by the modelling term based on the resolved modes. The noise amplitude $\epsilon$ should therefore be proportional to the intensity of the modelled term $T_{n}$ (equation (4.5)) and to the ratio $r$ of the high-frequency to the low-frequency component of the unresolved terms. It seems reasonable to assume that this ratio $r$ is of the order of the fraction of energy left in the higher-order modes: $r \sim 0.4$. The typical modelled term $T_{n}$ is of the order of a few $10^{-3}$ (for the largest fluctuating modes, it is about $210^{-3}$ ). This means that the noise level should be of the order of $\epsilon \sim r T_{n} \sim 10^{-3}$.

\section{REFERENCES}

BAI, K., Ji, D. \& Brown, E. 2016 Ability of a low-dimensional model to predict geometrydependent dynamics of large-scale coherent structures in turbulence. Physical Review E 93, 023117.

Bailon-Cuba, J., Emran, M. S. \& Schumacher, J. 2010 Aspect ratio dependece of heat transfer and large-scale flow in turbulent convection. Journal of Fluid Mechanics 655, $152-173$.

Benzi, R. \& Verzicco, R. 2008 Numerical simulations of flow reversal in Rayleigh-Bénard convection. Europhysics Letters 80 (64008).

Berkooz, G., Holmes, P. \& Lumley, J. L. 1993 The proper orthogonal decomposition in the analysis of turbulent flows. Annual Review of Fluid Mechanics 25, 539-575.

Brown, E. \& Ahlers, G. 2007 Large-scale circulation model for turbulent Rayleigh-Bénard convection. Physical Review Letters 98, 134501.

Brown, E., Nikolaenko, A. \& Ahlers, G. 2005 Reorientation of the large-scale circulation in turbulent Rayleigh-Bénard convection. Physical Review Letters 95, 084503. 
Castillo-Castellanos, A., Sergent, A. \& Rossi, M. 2016 Reversal cycle in square RayleighBénard cells in turbulent regime. Journal of Fluid Mechanics 808, 614-640.

Chandra, M. \& Verma, M. K. 2011 Dynamics and symmetries of flow reversals in turbulent convection. Physical Review E 83, 067303.

Chandra, M. \& Verma, M. K. 2013 Flow reversals in turbulent convection via vortex reconnections. Physical Review Letters 110, 114503.

Foroozani, N., Niemela, J. J., Armenio, V. \& Sreenivasan, K. R. 2017 Reorientations of the large-scale flow in turbulent convection in a cube. Physical Review E 95, 033107.

Giannakis, D., Kolchinskaya, A., Krasnov, D. \& Schumacher, J. 2018 Koopman analysis of the long-term evolution in a turbulent convection cell. Journal of Fluid Mechanics 847, $735-767$.

Holmes, P., Lumley, J. \& Berkooz, G. 1996 Turbulence, Coherent Structures, Dynamical Systems and Symmetry. Cambridge University Press.

Hunt, J. C. R., Wray, A. A. \& Moin, P. 1988 Eddies, streams and convergence zones in turbulent flows. In Center for Turbulence Research, Proceedings of the Summer Program.

Mishra, P. K., Verman, A. K. \& ESwaran, V. 2011 Dynamics of reorientations and reversals of large-scale flow in Rayleigh-Bénard convection. Journal of Fluid Mechanics 668, 480499.

Moin, P. \& Moser, R. D. 1989 Characteristic-eddy decomposition of turbulence in a channel. Journal of Fluid Mechanics 200, 471-509.

Podvin, B. \& Le QuéRÉ, P. 2001 Low-order models for the flow in a differentially heated cavity. Physics of Fluids 13 (11), 3204-3214.

Podvin, B. \& Sergent, A. 2012 Proper orthogonal decomposition investigation of turbulent Rayleigh-Bénard convection in a rectangular cavity. Physics of Fluids 24, 105106.

Podvin, B. \& Sergent, A. 2015 A large-scale investigation of wind reversal in a square Rayleigh-Bénard cell. Journal of Fluid Mechanics 766, 172-201.

Podvin, B. \& Sergent, A. 2017 Precursor for wind reversal in a square Rayleigh-Bénard cell. Physical Review E 95 (013112).

Puigjaner, D., Herrero, J., Simo, C. \& Giralt, F. 2008 Bifurcation analysis of steady Rayleigh-Bénard convection in a cubical cavity with conducting sidewalls. Journal of Fluid Mechanics 598, 393-427.

Rowley, C. W., Mezić, I., Bagheri, S., Schlatter, P. \& Henningson, D. S. 2009 Spectral analysis of nonlinear flows. Journal of Fluid Mechanics 641, 115-127.

Shishkina, O., Stevens, R. J. A. M., Grossmann, S. \& Lohse, D. 2010 Boundary layer structure in turbulent thermal convection and its consequences for the required numerical resolution. New Journal of Physics 12, 075022.

Sirovich, L. 1987 Turbulence and the dynamic of coherent structures. Part I: Coherent structures. Quarterly of Applied Mathematics 45 (3), 561-571.

Sreenivasan, K. R., Bershadski, A. \& Niemela, J. J. 2002 Mean wind and its reversal in thermal convection. Physical Review E 65 (056306).

Sugiyama, K., Ni, R., Stevens, R. J. A. M., Chan, T. S., Zhou, S.-Q., Xi, H.-D., Sun, C., Grossmann, S., Xia, K.-Q. \& Lohse, D. 2010 Flow reversals in thermally driven turbulence. Physical Review Letters 105, 034503.

Vasiliev, A., Frick, P., Kumar, A., Stepanov, R., Sukhanovskit, A. \& Verma, M. K. 2018 Mechanism of reorientations of turbulent large-scale convective flow in a cubic cell. Preprint, https://arxiv.org/abs/1805.06718.

Vasiliev, A., Sukhanovskit, A., Frick, P., Budnikov, A., Fomichev, V., Bolshukhin, M. \& Romanov, R. 2016 High Rayleigh number convection in a cubic cell with adiabatic sidewalls. International Journal of Heat and Mass Transfer 102, 201-212.

Verdoold, J., Tummers, M. J. \& Hanjalić, K. 2009 Prime modes of fluid circulation in large-aspect-ratio turbulent Rayleigh-Bénard convection. Physical Review E 80, 037301.

Xin, S., Chergui, J. \& Le Quéré, P. 2008 3D spectral parallel multi-domain computing for natural convection flows. In Parallel Computational Fluid Dynamics, pp. 163-171.

XIN, S. \& LE QuÉRÉ, P. 2002 An extended Chebyshev pseudo-spectral benchmark for the 8:1 differentially heated cavity. Int. J. Heat Mass Transfer 40, 981-998. 